\title{
POLARIZED LIGHT REFLECTED AND TRANSMITTED BY THICK RAYLEIGH SCATTERING ATMOSPHERES
}

\author{
ViJay Natraj ${ }^{1,3}$ and J. W. Hovenier ${ }^{2}$ \\ ${ }^{1}$ Jet Propulsion Laboratory, California Institute of Technology, Pasadena, CA 91109, USA; Vijay.Natraj@jpl.nasa.gov \\ 2 Astronomical Institute "Anton Pannekoek," University of Amsterdam, 1090 GE Amsterdam, The Netherlands \\ Received 2011 August 6; accepted 2011 December 12; published 2012 March 2
}

\begin{abstract}
Accurate values for the intensity and polarization of light reflected and transmitted by optically thick Rayleigh scattering atmospheres with a Lambert surface underneath are presented. A recently reported new method for solving integral equations describing Chandrasekhar's $X$ - and $Y$-functions is used. The results have been validated using various tests and techniques, including the doubling-adding method, and are accurate to within one unit in the eighth decimal place. Tables are stored electronically and expected to be useful as benchmark results for the (exo)planetary science and astrophysics communities. Asymptotic expressions to obtain Stokes parameters for a thick layer from those of a semi-infinite atmosphere are also provided.
\end{abstract}

Key words: methods: analytical - methods: numerical - planets and satellites: atmospheres - polarization radiative transfer - scattering

\section{INTRODUCTION}

Interesting information can be obtained from analyzing the polarization of light reflected and transmitted by planetary atmospheres. This is well known for solar system planets (see, e.g., Lyot 1929; Coffeen \& Hansen 1974; Hansen \& Hovenier 1974; van de Hulst 1980; West \& Smith 1991; Schmid et al. 2006a; Joos \& Schmid 2007). The discovery of hundreds of exoplanets in recent years (http://exoplanet.eu) has strongly promoted interest in high-accuracy polarimetry of such objects and several instruments are being developed for that purpose. At the same time model computations for the brightness and state of polarization of light reflected by exoplanets have been published to prepare for new observations and analyses of photometric and polarimetric data (see, e.g., Stam et al. 2004; Stam 2008; Buenzli $\&$ Schmid 2009). All of these developments have increased the need for easily accessible accurate benchmark numbers for a large range of optical thickness.

Light scattering by molecules and particles small compared to the wavelength, both inside and outside the particle, is commonly called Rayleigh scattering. It presents the simplest case for polarized light scattering by particles and is therefore an ideal test case for theoretical ideas and methods, as well as computations, for more complicated types of light scattering. In this paper, we only consider pure Rayleigh scattering, i.e., scattering by particles with isotropic polarizability, in a plane-parallel non-absorbing homogeneous atmosphere. Chandrasekhar (1950) was the first to develop a method for computing the intensity and state of polarization of light reflected and transmitted by such an atmosphere. This method was used by several authors (see, e.g., Sekera \& Blanch 1952; Chandrasekhar \& Elbert 1954; Coulson et al. 1960) to obtain results for atmospheres with optical thickness up to about unity.

Recently, Natraj et al. (2009) considerably improved the tables produced by Coulson et al. (1960) by using a new method and obtained results accurate to eight decimal places for several values of optical thickness in the range 0.02-1.0. This range is suitable for Earth's atmosphere in the visible

\footnotetext{
3 Author to whom any correspondence should be addressed.
}

part of the spectrum, but not for planetary atmospheres with an optically thick Rayleigh scattering layer. The importance of such atmospheres is gathering strength, since they are the best candidates for polarimetric detection of exoplanets (Schmid et al. 2006b).

Attempts to use Chandrasekhar's method for atmospheres with a finite optical thickness larger than unity ran into several kinds of problems. Various other methods were used to produce numerical results in the tabular form for the polarized light emerging from a non-absorbing plane-parallel Rayleigh scattering atmosphere (see, e.g., Dave \& Furukawa 1966; Adams \& Kattawar 1970; Fymat \& Abhyankar 1971). The results are, however, mostly of limited value due to their low accuracy and the small number of selected cases. Using a new method, Sekera \& Kahle (1966) generated tables of basic functions to five significant figures, from which the intensity and state of polarization of the reflected and transmitted light could be calculated for values of optical thickness in the range 0.15-100. However, even at an optical thickness of 100 the values have not yet reached the limiting values for a semi-infinite atmosphere to the same number of decimal places.

Chandrasekhar (1950) also considered the reflection of light by a semi-infinite homogeneous plane-parallel atmosphere in the context of Rayleigh scattering. He found a complete solution for the intensity and polarization of the reflected light in terms of several functions of one variable. These were tabulated to four or five decimal places; in addition, a few figures were presented of the angular dependence of the resulting Stokes parameters for incident unpolarized light. Bosma \& De Rooij (1983) proposed efficient methods to compute the basic functions for semiinfinite atmospheres and tabulated them to 10 decimal places including the case of conservative Rayleigh scattering. However, they neglected polarization in their analysis. De Rooij et al. (1989) extended this work to include polarization.

In this paper, we extend the work of Natraj et al. (2009) to optical thickness larger than unity. First, we generate tables of the intensity and polarization of light reflected and transmitted by Rayleigh scattering atmospheres with optical thickness unity and larger and a Lambert surface underneath. Second, we use the formulae and tables of Sekera \& Kahle (1966) to compute Stokes 
parameters and show that there is considerable inaccuracy in their results. Third, we provide Stokes parameter results for semi-infinite atmospheres and verify that we can reproduce the results for the basic functions in De Rooij et al. (1989). Fourth, we provide a means to transition from optically thick to semiinfinite atmospheres or vice versa using asymptotic theory. In this paper, thick refers to an optical thickness larger than or equal to unity.

Section 2 provides definitions for the Stokes parameters and other quantities of interest such as the degree and direction of polarization. Section 3 describes the computation of the Stokes parameters for atmospheres with infinite or large optical thickness. Some sample results are shown in Section 4. Section 5 illustrates the various methods that were used to validate the precision of the results. Conclusions are presented in Section 6.

\section{STOKES PARAMETERS AND GEOMETRY}

The main purpose of this section is to clarify the exact meaning of our tabulated quantities and to outline how to use these to obtain practical quantities of interest for experimental and observational purposes, such as the degree and direction of linear polarization. The intensity and state of polarization of a beam of quasi-monochromatic light traveling in one direction can be completely described by four Stokes parameters, $I, Q$, $U$, and $V$, where $I$ is the intensity, $Q$ and $U$ describe linearly polarized light, and $V$ refers to circularly polarized light (Stokes 1852). Several sign conventions for Stokes parameters can be found in the literature. As shown by Hovenier \& van der Mee (1983), special care is needed to establish the relationships among the sets of polarization parameters used by different authors. In this paper, we use the same definitions of Stokes parameters as given by Hovenier \& van der Mee (1983) and Hovenier et al. (2004), to which we refer for a more extensive treatment. These Stokes parameters are essentially the same as those in Chandrasekhar (1950) and van de Hulst (1957, 1980).

In this paper, we consider the reflection and transmission by a plane-parallel non-absorbing homogeneous atmosphere that is illuminated at the top by a parallel beam of unpolarized light with net flux $\pi F_{0}$ per unit area perpendicular to the incident beam. For all computations $F_{0}$ was taken to be unity. The light in the atmosphere is solely interacting by means of pure Rayleigh scattering with molecules or particles much smaller than the wavelength. Thus, the albedo of single scattering is unity and there is no depolarization. Under these conditions, the Stokes parameter $V$ of the reflected and transmitted light is identically zero and will not be considered hereafter. Therefore, only linear polarization occurs for the reflected and transmitted light.

The geometry of the problem is illustrated in Figure 1. A point $\mathrm{O}$ of a homogeneous plane-parallel atmosphere is the origin of a Cartesian coordinate system defined by the ordered triplet $(x$, $y, z$ ) of axes, where the positive $z$-axis is the local vertical of the atmosphere. Suppose a light beam is traveling in the direction OP. The points $\mathrm{P}$ and $\mathrm{N}$ are located on a unit sphere. $\mathrm{O}, \mathrm{P}$, and $\mathrm{N}$ lie in one plane, called the meridian plane. At $\mathrm{P}$ we choose unit vectors $\mathbf{I}$ and $\mathbf{r}$ in the plane perpendicular to OP in such a way that (1) $\mathbf{I}$ lies in the meridian plane and $\mathbf{r}$ is perpendicular to this plane, and (2) the direction of the vector product $\mathbf{r} \times \mathbf{l}$ is the same as the direction of propagation of the beam, i.e., OP. Suppose the light traveling in the direction $\mathrm{OP}$ is linearly polarized along a line making an angle $\chi$ with the positive l-axis. This angle is obtained by rotating $\mathbf{l}$ in the counterclockwise direction when

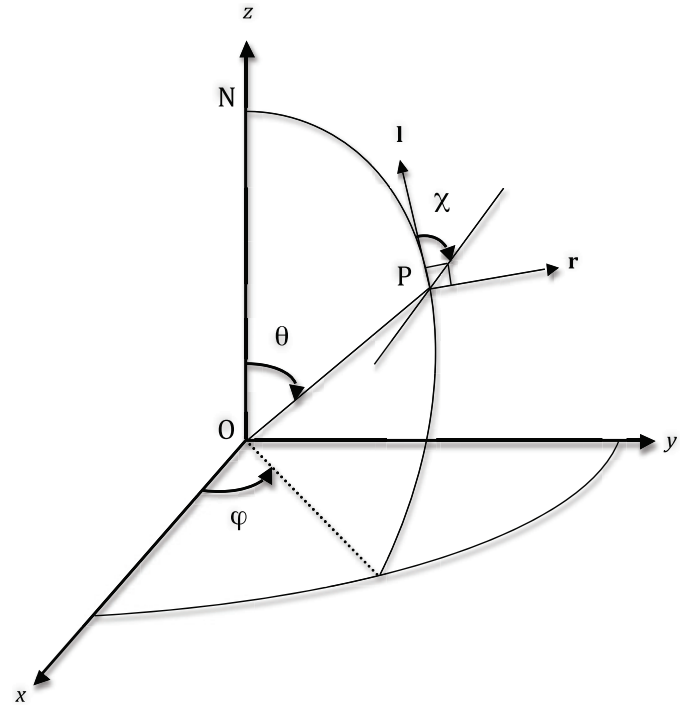

Figure 1. Geometry of a beam of linearly polarized light traveling in the direction $\mathrm{OP}$.

looking in the direction of propagation until $\mathbf{l}$ is directed along the direction of the linearly polarized light. The angle $\chi$ always lies in the range $0 \leqslant \chi<\pi$.

The direction of OP is specified by the polar angle $\theta$ and azimuthal angle $\varphi$, which is measured clockwise when looking from bottom to top in the atmosphere starting from a chosen horizontal direction, which is the positive $x$-direction in Figure 1. Since we consider a horizontally homogeneous atmosphere, only the azimuth difference of the outgoing and incident directions is relevant. For convenience, we will take the azimuth angle of the incident light to be zero.

Instead of the polar angle, we generally use for points at the top (TOA) and bottom (BOA) of the atmosphere $\mu$, which is the absolute value of $\cos \theta$. Furthermore, instead of the polar angle of the unidirectional beam incident at the top, we use $\mu_{0}$, which is the absolute value of the cosine of its polar angle. Thus, the direction of the emergent light is given by the pair $(\mu, \varphi)$ and that of the incident light by $\left(\mu_{0}, 0\right)$. When light is incident $\left(\mu_{0}=1\right)$ or emergent $(\mu=1)$ in a perpendicular direction, there is no unique meridian plane. In these cases, we can first choose a particular meridian plane and then let $\mu_{0}$ (for perpendicular incidence) or $\mu$ (for perpendicular emergence) tend to 1 in that plane (Hovenier \& de Haan 1985).

The tables of Stokes parameters of the reflected and transmitted light presented in this paper can be used to obtain several quantities that are important for physical understanding of the scattering processes and also for experimental and observational purposes. For example, the degree of (linear) polarization is

$$
p=\frac{\sqrt{Q^{2}+U^{2}}}{I},
$$

which is zero for unpolarized light and unity for fully polarized light.

However, in case $U$ vanishes it is often handy to use:

$$
p_{s}=-Q / I \text {, }
$$

which is positive when the vibrations in the r-direction dominate over those in the l-direction, and negative in the opposite case. This convention is very common, especially in astronomy. 
Table 1

$I$ (Reflection at TOA) for $b=16, \mu_{0}=0.2$, and $A=0$

\begin{tabular}{|c|c|c|c|c|c|c|c|}
\hline$\mu$ & $\varphi=0^{\circ}$ & $\varphi=30^{\circ}$ & $\varphi=60^{\circ}$ & $\varphi=90^{\circ}$ & $\varphi=120^{\circ}$ & $\varphi=150^{\circ}$ & $\varphi=180^{\circ}$ \\
\hline 0.00 & 0.50563312 & 0.45408360 & 0.35098455 & 0.29943502 & 0.35098455 & 0.45408360 & 0.50563312 \\
\hline 0.02 & 0.48479002 & 0.43707859 & 0.34181798 & 0.29471204 & 0.34347221 & 0.43994381 & 0.48809849 \\
\hline 0.04 & 0.46180870 & 0.41756203 & 0.32937337 & 0.28626374 & 0.33247983 & 0.42294257 & 0.46802161 \\
\hline 0.06 & 0.44047780 & 0.39928264 & 0.31732257 & 0.27773308 & 0.32170933 & 0.40688073 & 0.44925131 \\
\hline 0.08 & 0.42104596 & 0.38255321 & 0.30610938 & 0.26963819 & 0.31163238 & 0.39211932 & 0.43209195 \\
\hline 0.10 & 0.40338851 & 0.36730819 & 0.29578872 & 0.26210131 & 0.30232627 & 0.37863156 & 0.41646361 \\
\hline 0.12 & 0.38730853 & 0.35339917 & 0.28631094 & 0.25512779 & 0.29375909 & 0.36629975 & 0.40220484 \\
\hline 0.14 & 0.37260948 & 0.34066861 & 0.27759784 & 0.24868360 & 0.28586673 & 0.35499075 & 0.38914726 \\
\hline 0.16 & 0.35911472 & 0.32897117 & 0.26956784 & 0.24272257 & 0.27857889 & 0.34457876 & 0.37713681 \\
\hline 0.18 & 0.34667119 & 0.31817910 & 0.26214467 & 0.23719707 & 0.27182841 & 0.33495185 & 0.36603869 \\
\hline 0.20 & 0.33514812 & 0.30818220 & 0.25526002 & 0.23206213 & 0.26555445 & 0.32601269 & 0.35573698 \\
\hline 0.22 & 0.32443421 & 0.29888609 & 0.24885391 & 0.22727689 & 0.25970314 & 0.31767750 & 0.34613265 \\
\hline 0.24 & 0.31443477 & 0.29021005 & 0.24287409 & 0.22280493 & 0.25422729 & 0.30987436 & 0.33714116 \\
\hline 0.26 & 0.30506905 & 0.28208498 & 0.23727518 & 0.21861408 & 0.24908575 & 0.30254149 & 0.32869019 \\
\hline 0.30 & 0.28797202 & 0.26725866 & 0.22706760 & 0.21096585 & 0.23966677 & 0.28908106 & 0.31317037 \\
\hline 0.32 & 0.28013011 & 0.26046211 & 0.22239482 & 0.20746169 & 0.23533072 & 0.28286775 & 0.30600192 \\
\hline 0.34 & 0.27269782 & 0.25402358 & 0.21797334 & 0.20414425 & 0.23121056 & 0.27695112 & 0.29917227 \\
\hline 0.36 & 0.26563656 & 0.24790963 & 0.21378029 & 0.20099651 & 0.22728523 & 0.27130087 & 0.29264644 \\
\hline 0.38 & 0.25891264 & 0.24209108 & 0.20979558 & 0.19800341 & 0.22353614 & 0.26589044 & 0.28639377 \\
\hline 0.40 & 0.25249653 & 0.23654227 & 0.20600148 & 0.19515159 & 0.21994686 & 0.26069639 & 0.28038730 \\
\hline 0.42 & 0.24636228 & 0.23124067 & 0.20238232 & 0.19242915 & 0.21650278 & 0.25569801 & 0.27460319 \\
\hline 0.44 & 0.24048702 & 0.22616635 & 0.19892422 & 0.18982550 & 0.21319086 & 0.25087689 & 0.26902030 \\
\hline 0.46 & 0.23485049 & 0.22130167 & 0.19561483 & 0.18733115 & 0.20999947 & 0.24621661 & 0.26361978 \\
\hline 0.48 & 0.22943477 & 0.21663101 & 0.19244314 & 0.18493760 & 0.20691814 & 0.24170246 & 0.25838478 \\
\hline 0.50 & 0.22422391 & 0.21214044 & 0.18939934 & 0.18263718 & 0.20393745 & 0.23732120 & 0.25330015 \\
\hline 0.52 & 0.21920375 & 0.20781759 & 0.18647464 & 0.18042302 & 0.20104887 & 0.23306089 & 0.24835220 \\
\hline 0.54 & 0.21436166 & 0.20365141 & 0.18366120 & 0.17828887 & 0.19824465 & 0.22891068 & 0.24352855 \\
\hline 0.56 & 0.20968639 & 0.19963207 & 0.18095198 & 0.17622909 & 0.19551772 & 0.22486069 & 0.23881789 \\
\hline 0.58 & 0.20516793 & 0.19575078 & 0.17834065 & 0.17423855 & 0.19286163 & 0.22090185 & 0.23420988 \\
\hline 0.64 & 0.19246915 & 0.18486139 & 0.17104055 & 0.16863780 & 0.18526089 & 0.20949174 & 0.22090983 \\
\hline 0.66 & 0.18849843 & 0.18146258 & 0.16877009 & 0.16688150 & 0.18283266 & 0.20581965 & 0.21662355 \\
\hline 0.68 & 0.18464928 & 0.17817088 & 0.16657483 & 0.16517483 & 0.18044928 & 0.20220213 & 0.21239818 \\
\hline 0.70 & 0.18091718 & 0.17498229 & 0.16445171 & 0.16351479 & 0.17810642 & 0.19863295 & 0.20822661 \\
\hline 0.72 & 0.17729841 & 0.17189351 & 0.16239811 & 0.16189860 & 0.17579988 & 0.19510606 & 0.20410195 \\
\hline 0.74 & 0.17379001 & 0.16890192 & 0.16041189 & 0.16032374 & 0.17352556 & 0.19161547 & 0.20001735 \\
\hline 0.76 & 0.17038989 & 0.16600564 & 0.15849136 & 0.15878785 & 0.17127936 & 0.18815512 & 0.19596590 \\
\hline 0.78 & 0.16709687 & 0.16320360 & 0.15663533 & 0.15728875 & 0.16905713 & 0.18471878 & 0.19194047 \\
\hline 0.80 & 0.16391087 & 0.16049565 & 0.15484322 & 0.15582444 & 0.16685453 & 0.18129985 & 0.18793349 \\
\hline 0.82 & 0.16083308 & 0.15788277 & 0.15311509 & 0.15439303 & 0.16466689 & 0.17789107 & 0.18393668 \\
\hline 0.84 & 0.15786637 & 0.15536739 & 0.15145191 & 0.15299280 & 0.16248903 & 0.17448424 & 0.17994061 \\
\hline 0.86 & 0.15501585 & 0.15295390 & 0.14985579 & 0.15162213 & 0.16031489 & 0.17106959 & 0.17593405 \\
\hline 0.88 & 0.15228991 & 0.15064951 & 0.14833049 & 0.15027952 & 0.15813702 & 0.16763491 & 0.17190297 \\
\hline 0.90 & 0.14970205 & 0.14846586 & 0.14688237 & 0.14896357 & 0.15594565 & 0.16416392 & 0.16782861 \\
\hline 0.92 & 0.14727445 & 0.14642212 & 0.14552215 & 0.14767295 & 0.15372687 & 0.16063312 & 0.16368390 \\
\hline 0.94 & 0.14504605 & 0.14455197 & 0.14426895 & 0.14640646 & 0.15145858 & 0.15700478 & 0.15942532 \\
\hline 0.96 & 0.14309449 & 0.14292261 & 0.14316126 & 0.14516293 & 0.14909950 & 0.15320794 & 0.15497096 \\
\hline 0.98 & 0.14162184 & 0.14170900 & 0.14229982 & 0.14394130 & 0.14654629 & 0.14906411 & 0.15011478 \\
\hline 1.00 & 0.14274056 & 0.14274056 & 0.14274056 & 0.14274056 & 0.14274056 & 0.14274056 & 0.14274056 \\
\hline
\end{tabular}

If $Q$ is not zero we can find the direction of the linearly polarized light from

$$
\tan 2 \chi=U / Q
$$

and

$$
\operatorname{sgn}(\cos 2 \chi)=\operatorname{sgn} Q,
$$

where sgn stands for "the sign of." Therefore, from the different values of $\chi$ differing by $\pi / 2$ that satisfy Equation (3) we must choose the value that satisfies Equation (4).
If $Q$ is zero and $U$ is nonzero, Equation (3) shows that $\chi$ equals $\pi / 4$ or $3 \pi / 4$, but we know that $\chi=\pi / 4$ if $U>0$ and $\chi=3 \pi / 4$ if $U<0$, since

$$
\operatorname{sgn}(\sin 2 \chi)=\operatorname{sgn} U .
$$

If $Q=U=0$, Equation (1) shows that the light is unpolarized, so that there is no preferential direction for the vibrations of the light.

In addition to $I$ and $Q$, we will also use the intensity $I_{l}$ in the l-direction and the intensity $I_{r}$ in the $\mathbf{r}$-direction. The 
Table 2

$I$ (Transmission at BOA) for $b=16, \mu_{0}=0.2$, and $A=0$

\begin{tabular}{|c|c|c|c|c|c|c|c|}
\hline$\mu$ & $\varphi=0^{\circ}$ & $\varphi=30^{\circ}$ & $\varphi=60^{\circ}$ & $\varphi=90^{\circ}$ & $\varphi=120^{\circ}$ & $\varphi=150^{\circ}$ & $\varphi=180^{\circ}$ \\
\hline 0.00 & 0.00389667 & 0.00389665 & 0.00389659 & 0.00389656 & 0.00389659 & 0.00389665 & 0.00389667 \\
\hline 0.02 & 0.00415558 & 0.00415555 & 0.00415549 & 0.00415546 & 0.00415549 & 0.00415555 & 0.00415558 \\
\hline 0.04 & 0.00436584 & 0.00436580 & 0.00436574 & 0.00436571 & 0.00436574 & 0.00436580 & 0.00436584 \\
\hline 0.06 & 0.00456077 & 0.00456073 & 0.00456067 & 0.00456064 & 0.00456067 & 0.00456073 & 0.00456077 \\
\hline 0.08 & 0.00474681 & 0.00474678 & 0.00474671 & 0.00474668 & 0.00474671 & 0.00474678 & 0.00474681 \\
\hline 0.10 & 0.00492685 & 0.00492681 & 0.00492675 & 0.00492671 & 0.00492675 & 0.00492681 & 0.00492685 \\
\hline 0.14 & 0.00527473 & 0.00527469 & 0.00527462 & 0.00527459 & 0.00527462 & 0.00527469 & 0.00527473 \\
\hline 0.16 & 0.00544427 & 0.00544423 & 0.00544416 & 0.00544412 & 0.00544416 & 0.00544423 & 0.00544427 \\
\hline 0.18 & 0.00561160 & 0.00561156 & 0.00561149 & 0.00561145 & 0.00561149 & 0.00561156 & 0.00561160 \\
\hline 0.20 & 0.00577708 & 0.00577705 & 0.00577697 & 0.00577693 & 0.00577697 & 0.00577704 & 0.00577708 \\
\hline 0.22 & 0.00594100 & 0.00594097 & 0.00594089 & 0.00594085 & 0.00594089 & 0.00594096 & 0.00594100 \\
\hline 0.24 & 0.00610358 & 0.00610354 & 0.00610346 & 0.00610342 & 0.00610346 & 0.00610354 & 0.00610358 \\
\hline 0.26 & 0.00626499 & 0.00626495 & 0.00626487 & 0.00626483 & 0.00626487 & 0.00626495 & 0.00626499 \\
\hline 0.30 & 0.00658487 & 0.00658483 & 0.00658475 & 0.00658471 & 0.00658475 & 0.00658483 & 0.00658487 \\
\hline 0.32 & 0.00674356 & 0.00674352 & 0.00674343 & 0.00674339 & 0.00674343 & 0.00674351 & 0.00674356 \\
\hline 0.34 & 0.00690153 & 0.00690149 & 0.00690140 & 0.00690136 & 0.00690140 & 0.00690148 & 0.00690153 \\
\hline 0.36 & 0.00705885 & 0.00705881 & 0.00705873 & 0.00705868 & 0.00705873 & 0.00705881 & 0.00705885 \\
\hline 0.38 & 0.00721560 & 0.00721556 & 0.00721547 & 0.00721543 & 0.00721547 & 0.00721555 & 0.00721559 \\
\hline 0.40 & 0.00737181 & 0.00737177 & 0.00737168 & 0.00737164 & 0.00737168 & 0.00737177 & 0.00737181 \\
\hline 0.42 & 0.00752755 & 0.00752750 & 0.00752742 & 0.00752737 & 0.00752741 & 0.00752750 & 0.00752754 \\
\hline 0.44 & 0.00768284 & 0.00768280 & 0.00768271 & 0.00768267 & 0.00768271 & 0.00768279 & 0.00768284 \\
\hline 0.46 & 0.00783773 & 0.00783769 & 0.00783760 & 0.00783755 & 0.00783760 & 0.00783768 & 0.00783773 \\
\hline 0.48 & 0.00799225 & 0.00799221 & 0.00799212 & 0.00799207 & 0.00799211 & 0.00799220 & 0.00799225 \\
\hline 0.50 & 0.00814643 & 0.00814638 & 0.00814629 & 0.00814625 & 0.00814629 & 0.00814638 & 0.00814642 \\
\hline 0.52 & 0.00830029 & 0.00830024 & 0.00830015 & 0.00830011 & 0.00830015 & 0.00830024 & 0.00830028 \\
\hline 0.54 & 0.00845385 & 0.00845381 & 0.00845372 & 0.00845367 & 0.00845371 & 0.00845380 & 0.00845384 \\
\hline 0.56 & 0.00860714 & 0.00860710 & 0.00860701 & 0.00860696 & 0.00860701 & 0.00860709 & 0.00860714 \\
\hline 0.58 & 0.00876018 & 0.00876014 & 0.00876005 & 0.00876000 & 0.00876004 & 0.00876013 & 0.00876017 \\
\hline 0.64 & 0.00921793 & 0.00921789 & 0.00921780 & 0.00921775 & 0.00921780 & 0.00921788 & 0.00921792 \\
\hline 0.66 & 0.00937011 & 0.00937006 & 0.00936998 & 0.00936993 & 0.00936997 & 0.00937005 & 0.00937010 \\
\hline 0.68 & 0.00952210 & 0.00952206 & 0.00952197 & 0.00952193 & 0.00952197 & 0.00952205 & 0.00952209 \\
\hline 0.70 & 0.00967392 & 0.00967388 & 0.00967379 & 0.00967375 & 0.00967379 & 0.00967387 & 0.00967391 \\
\hline 0.72 & 0.00982558 & 0.00982554 & 0.00982545 & 0.00982541 & 0.00982545 & 0.00982552 & 0.00982556 \\
\hline 0.74 & 0.00997708 & 0.00997704 & 0.00997696 & 0.00997692 & 0.00997695 & 0.00997703 & 0.00997707 \\
\hline 0.76 & 0.01012844 & 0.01012840 & 0.01012832 & 0.01012828 & 0.01012832 & 0.01012839 & 0.01012843 \\
\hline 0.78 & 0.01027966 & 0.01027962 & 0.01027955 & 0.01027951 & 0.01027954 & 0.01027961 & 0.01027965 \\
\hline 0.80 & 0.01043075 & 0.01043072 & 0.01043065 & 0.01043061 & 0.01043064 & 0.01043070 & 0.01043074 \\
\hline 0.82 & 0.01058172 & 0.01058169 & 0.01058162 & 0.01058158 & 0.01058161 & 0.01058167 & 0.01058171 \\
\hline 0.84 & 0.01073257 & 0.01073254 & 0.01073248 & 0.01073244 & 0.01073247 & 0.01073253 & 0.01073256 \\
\hline 0.86 & 0.01088331 & 0.01088329 & 0.01088322 & 0.01088319 & 0.01088322 & 0.01088327 & 0.01088330 \\
\hline 0.88 & 0.01103395 & 0.01103392 & 0.01103387 & 0.01103384 & 0.01103386 & 0.01103391 & 0.01103393 \\
\hline 0.90 & 0.01118448 & 0.01118446 & 0.01118441 & 0.01118438 & 0.01118440 & 0.01118444 & 0.01118446 \\
\hline 0.92 & 0.01133492 & 0.01133490 & 0.01133486 & 0.01133483 & 0.01133485 & 0.01133488 & 0.01133490 \\
\hline 0.94 & 0.01148526 & 0.01148525 & 0.01148521 & 0.01148519 & 0.01148520 & 0.01148523 & 0.01148525 \\
\hline 0.96 & 0.01163552 & 0.01163551 & 0.01163548 & 0.01163547 & 0.01163547 & 0.01163549 & 0.01163550 \\
\hline 0.98 & 0.01178569 & 0.01178568 & 0.01178567 & 0.01178566 & 0.01178566 & 0.01178567 & 0.01178568 \\
\hline 1.00 & 0.01193577 & 0.01193577 & 0.01193577 & 0.01193577 & 0.01193577 & 0.01193577 & 0.01193577 \\
\hline
\end{tabular}

relationships are

$$
\begin{aligned}
& I=I_{l}+I_{r} ; \\
& Q=I_{l}-I_{r} .
\end{aligned}
$$

For a Rayleigh scattering atmosphere, we can write for the light emerging at the top (Hovenier et al. 2004):

$$
\begin{aligned}
I_{l}(0 ; \mu, \varphi)= & I_{l}^{0}\left(0 ; \mu, \mu_{0}\right)+I_{l}^{1}\left(0 ; \mu, \mu_{0}\right) \cos \varphi \\
& +I_{l}^{2}\left(0 ; \mu, \mu_{0}\right) \cos 2 \varphi ;
\end{aligned}
$$

$$
\begin{aligned}
I_{r}(0 ; \mu, \varphi)= & I_{r}^{0}\left(0 ; \mu, \mu_{0}\right)+I_{r}^{1}\left(0 ; \mu, \mu_{0}\right) \cos \varphi \\
& +I_{r}^{2}\left(0 ; \mu, \mu_{0}\right) \cos 2 \varphi ;
\end{aligned}
$$

$$
U(0 ; \mu, \varphi)=U^{1}\left(0 ; \mu, \mu_{0}\right) \sin \varphi+U^{2}\left(0 ; \mu, \mu_{0}\right) \sin 2 \varphi,
$$

where superscripts 0,1 , and 2 are used to number the Fourier components and subscripts $l$ and $r$ refer to the two components of the intensity.

A similar Fourier decomposition is obtained for light emerging at the bottom by replacing zero by $b$ as the first variable in 
Table 3

$Q$ (Reflection at TOA) for $b=16, \mu_{0}=0.2$, and $A=0$

\begin{tabular}{|c|c|c|c|c|c|c|c|}
\hline$\mu$ & $\varphi=0^{\circ}$ & $\varphi=30^{\circ}$ & $\varphi=60^{\circ}$ & $\varphi=90^{\circ}$ & $\varphi=120^{\circ}$ & $\varphi=150^{\circ}$ & $\varphi=180^{\circ}$ \\
\hline 0.00 & 0.01203139 & 0.06358091 & 0.16667996 & 0.21822949 & 0.16667996 & 0.06358091 & 0.01203139 \\
\hline 0.02 & 0.01642270 & 0.06461574 & 0.16116407 & 0.20996260 & 0.16281830 & 0.06748096 & 0.01973117 \\
\hline 0.04 & 0.01744233 & 0.06266452 & 0.15341357 & 0.19977280 & 0.15652003 & 0.06804506 & 0.02365525 \\
\hline 0.06 & 0.01734043 & 0.06001294 & 0.14578819 & 0.19006636 & 0.15017494 & 0.06761102 & 0.02611394 \\
\hline 0.08 & 0.01662574 & 0.05710378 & 0.13860154 & 0.18110114 & 0.14412454 & 0.06666989 & 0.02767172 \\
\hline 0.10 & 0.01553489 & 0.05411354 & 0.13191200 & 0.17288356 & 0.13844955 & 0.06543691 & 0.02861000 \\
\hline 0.14 & 0.01269966 & 0.04817759 & 0.11994442 & 0.15844896 & 0.12821331 & 0.06249973 & 0.02923745 \\
\hline 0.16 & 0.01108733 & 0.04529272 & 0.11458725 & 0.15209091 & 0.12359830 & 0.06090031 & 0.02910943 \\
\hline 0.18 & 0.00939737 & 0.04247921 & 0.10959263 & 0.14621897 & 0.11927638 & 0.05925196 & 0.02876487 \\
\hline 0.20 & 0.00765402 & 0.03974041 & 0.10492282 & 0.14077724 & 0.11521726 & 0.05757089 & 0.02824288 \\
\hline 0.22 & 0.00587450 & 0.03707635 & 0.10054411 & 0.13571706 & 0.11139334 & 0.05586776 & 0.02757295 \\
\hline 0.24 & 0.00407133 & 0.03448531 & 0.09642677 & 0.13099631 & 0.10777996 & 0.05414962 & 0.02677771 \\
\hline 0.26 & 0.00225373 & 0.03196461 & 0.09254470 & 0.12657855 & 0.10435527 & 0.05242111 & 0.02587487 \\
\hline 0.30 & -0.00139875 & 0.02712159 & 0.08539795 & 0.11852990 & 0.09799712 & 0.04894399 & 0.02379959 \\
\hline 0.32 & -0.00322434 & 0.02479278 & 0.08209573 & 0.11484772 & 0.09503163 & 0.04719842 & 0.02264746 \\
\hline 0.34 & -0.00504499 & 0.02252160 & 0.07895304 & 0.11136479 & 0.09219026 & 0.04544914 & 0.02142945 \\
\hline 0.36 & -0.00685823 & 0.02030511 & 0.07595632 & 0.10806281 & 0.08946125 & 0.04369635 & 0.02015164 \\
\hline 0.38 & -0.00866206 & 0.01814062 & 0.07309359 & 0.10492566 & 0.08683416 & 0.04193998 & 0.01881908 \\
\hline 0.40 & -0.01045483 & 0.01602563 & 0.07035427 & 0.10193909 & 0.08429965 & 0.04017974 & 0.01743594 \\
\hline 0.42 & -0.01223521 & 0.01395788 & 0.06772895 & 0.09909048 & 0.08184940 & 0.03841522 & 0.01600570 \\
\hline 0.44 & -0.01400203 & 0.01193533 & 0.06520927 & 0.09636858 & 0.07947591 & 0.03664588 & 0.01453125 \\
\hline 0.46 & -0.01575428 & 0.00995615 & 0.06278780 & 0.09376338 & 0.07717245 & 0.03487109 & 0.01301501 \\
\hline 0.48 & -0.01749105 & 0.00801871 & 0.06045790 & 0.09126588 & 0.07493291 & 0.03309016 & 0.01145897 \\
\hline 0.50 & -0.01921150 & 0.00612160 & 0.05821362 & 0.08886804 & 0.07275174 & 0.03130235 & 0.00986474 \\
\hline 0.52 & -0.02091481 & 0.00426356 & 0.05604967 & 0.08656258 & 0.07062390 & 0.02950686 & 0.00823364 \\
\hline 0.54 & -0.02260018 & 0.00244355 & 0.05396131 & 0.08434295 & 0.06854475 & 0.02770282 & 0.00656671 \\
\hline 0.56 & -0.02426680 & 0.00066071 & 0.05194428 & 0.08220322 & 0.06651002 & 0.02588933 & 0.00486470 \\
\hline 0.58 & -0.02591380 & -0.00108566 & 0.04999480 & 0.08013799 & 0.06451577 & 0.02406541 & 0.00312815 \\
\hline 0.64 & -0.03072761 & -0.00611002 & 0.04451984 & 0.07434243 & 0.05874018 & 0.01852033 & -0.00228693 \\
\hline 0.66 & -0.03228611 & -0.00771363 & 0.04281055 & 0.07253029 & 0.05687311 & 0.01664345 & -0.00416099 \\
\hline 0.68 & -0.03381936 & -0.00928130 & 0.04115556 & 0.07077202 & 0.05503001 & 0.01474995 & -0.00607046 \\
\hline 0.70 & -0.03532573 & -0.01081248 & 0.03955324 & 0.06906447 & 0.05320795 & 0.01283818 & -0.00801630 \\
\hline 0.72 & -0.03680335 & -0.01230628 & 0.03800225 & 0.06740471 & 0.05140402 & 0.01090627 & -0.00999981 \\
\hline 0.74 & -0.03825000 & -0.01376150 & 0.03650164 & 0.06579008 & 0.04961531 & 0.00895204 & -0.01202266 \\
\hline 0.76 & -0.03966304 & -0.01517649 & 0.03505080 & 0.06421808 & 0.04783880 & 0.00697298 & -0.01408703 \\
\hline 0.78 & -0.04103926 & -0.01654909 & 0.03364955 & 0.06268642 & 0.04607135 & 0.00496610 & -0.01619567 \\
\hline 0.80 & -0.04237475 & -0.01787643 & 0.03229821 & 0.06119297 & 0.04430952 & 0.00292776 & -0.01835213 \\
\hline 0.82 & -0.04366456 & -0.01915479 & 0.03099772 & 0.05973575 & 0.04254952 & 0.00085352 & -0.02056096 \\
\hline 0.84 & -0.04490240 & -0.02037915 & 0.02974981 & 0.05831292 & 0.04078693 & -0.00126230 & -0.02282816 \\
\hline 0.86 & -0.04607994 & -0.02154278 & 0.02855731 & 0.05692277 & 0.03901642 & -0.00342709 & -0.02516173 \\
\hline 0.88 & -0.04718583 & -0.02263626 & 0.02742468 & 0.05556369 & 0.03723120 & -0.00565085 & -0.02757277 \\
\hline 0.90 & -0.04820382 & -0.02364589 & 0.02635887 & 0.05423419 & 0.03542215 & -0.00794783 & -0.03007726 \\
\hline 0.92 & -0.04910916 & -0.02455061 & 0.02537119 & 0.05293288 & 0.03357591 & -0.01033961 & -0.03269972 \\
\hline 0.94 & -0.04986052 & -0.02531496 & 0.02448130 & 0.05165846 & 0.03167094 & -0.01286215 & -0.03548126 \\
\hline 0.96 & -0.05037803 & -0.02587009 & 0.02372820 & 0.05040968 & 0.02966643 & -0.01558476 & -0.03850155 \\
\hline 0.98 & -0.05045752 & -0.02603948 & 0.02321306 & 0.04918542 & 0.02745954 & -0.01868438 & -0.04196458 \\
\hline 1.00 & -0.04798457 & -0.02399229 & 0.02399229 & 0.04798457 & 0.02399229 & -0.02399229 & -0.04798457 \\
\hline
\end{tabular}

the functions above, where $b$ stands for the optical thickness of the atmosphere. The azimuth independent and dependent terms in Equations (7) are, for finite optical thickness, related to the $X$ - and $Y$-functions and their moments (Chandrasekhar \& Elbert 1954). The readers are referred to Natraj et al. (2009) for details.

\section{EXPRESSIONS FOR INFINITE AND LARGE OPTICAL THICKNESS}

In this section, we first consider the special case of reflected light emerging from the top of an atmosphere with infinite optical thickness. Such an atmosphere has no bottom so transmission does not exist. The reflection can be expressed as follows (Chandrasekhar 1950):

$$
\begin{aligned}
\left(\begin{array}{l}
I_{l, \infty}^{0}\left(0 ; \mu, \mu_{0}\right) \\
I_{r, \infty}^{0}\left(0 ; \mu, \mu_{0}\right)
\end{array}\right)= & \frac{C}{\mu+\mu_{0}} \mathbf{A}(\mu) \mathbf{A}^{T}\left(\mu_{0}\right)\left(\begin{array}{l}
1 \\
1
\end{array}\right) ; \\
I_{l, \infty}^{1}\left(0 ; \mu, \mu_{0}\right)= & -\frac{4 C}{\mu+\mu_{0}} \mu \mu_{0}\left(1-\mu^{2}\right)^{1 / 2} \\
& \times\left(1-\mu_{0}^{2}\right)^{1 / 2} M^{1}\left(\mu, \mu_{0}\right) ;
\end{aligned}
$$


Table 4

$Q$ (Transmission at BOA) for $b=16, \mu_{0}=0.2$, and $A=0$

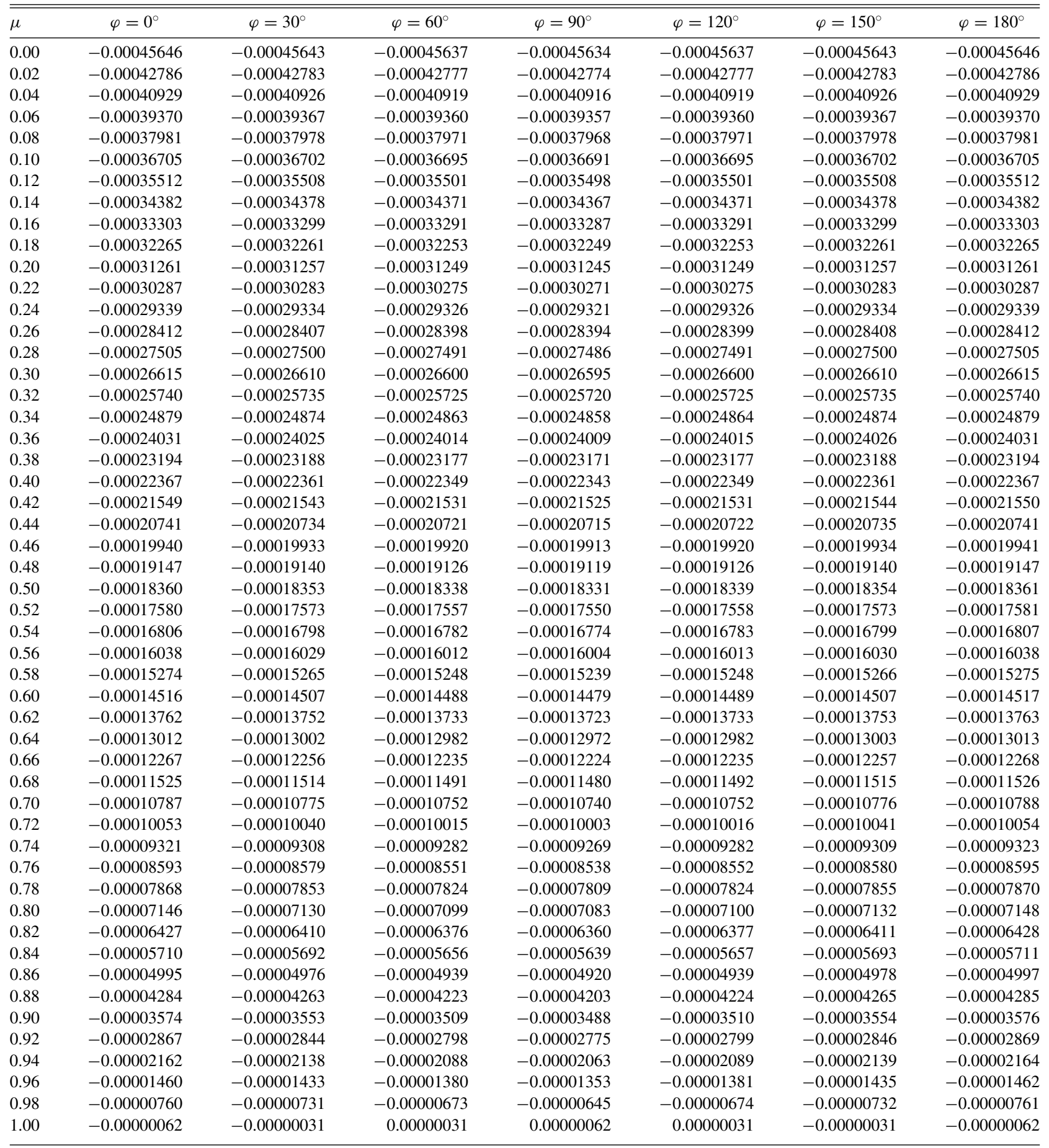

$$
I_{r, \infty}^{1}\left(0 ; \mu, \mu_{0}\right)=0
$$

$$
\begin{aligned}
U_{\infty}^{1}\left(0 ; \mu, \mu_{0}\right)= & -\frac{4 C}{\mu+\mu_{0}} \mu_{0}\left(1-\mu^{2}\right)^{1 / 2} \\
& \times\left(1-\mu_{0}^{2}\right)^{1 / 2} M^{1}\left(\mu, \mu_{0}\right) ;
\end{aligned}
$$

$I_{l, \infty}^{2}\left(0 ; \mu, \mu_{0}\right)=-\frac{C}{\mu+\mu_{0}} \mu^{2}\left(1-\mu_{0}^{2}\right) M^{2}\left(\mu, \mu_{0}\right)$

$$
\begin{array}{r}
I_{r, \infty}^{2}\left(0 ; \mu, \mu_{0}\right)=\frac{C}{\mu+\mu_{0}}\left(1-\mu_{0}^{2}\right) M^{2}\left(\mu, \mu_{0}\right) ; \\
U_{\infty}^{2}\left(0 ; \mu, \mu_{0}\right)=-\frac{2 C}{\mu+\mu_{0}} \mu\left(1-\mu_{0}^{2}\right) M^{2}\left(\mu, \mu_{0}\right),
\end{array}
$$

where $\mathbf{A}^{\mathrm{T}}$ means the transpose of $\mathbf{A}$, and the subscript $\infty$ implies that the atmosphere is semi-infinite. The constant $C$ is given by

$$
C=\frac{3}{32} \mu_{0} F_{0} .
$$


Table 5

$U$ (Reflection at TOA) for $b=16, \mu_{0}=0.2$, and $A=0$

\begin{tabular}{|c|c|c|c|c|c|c|c|}
\hline$\mu$ & $\varphi=0^{\circ}$ & $\varphi=30^{\circ}$ & $\varphi=60^{\circ}$ & $\varphi=90^{\circ}$ & $\varphi=120^{\circ}$ & $\varphi=150^{\circ}$ & $\varphi=180^{\circ}$ \\
\hline 0.00 & 0.00000000 & -0.04393411 & -0.07609611 & -0.08786822 & -0.07609611 & -0.04393411 & 0.00000000 \\
\hline 0.02 & 0.00000000 & -0.04467808 & -0.07495267 & -0.08271171 & -0.06830821 & -0.03803363 & 0.00000000 \\
\hline 0.04 & 0.00000000 & -0.04502929 & -0.07345535 & -0.07766142 & -0.06105818 & -0.03263213 & 0.00000000 \\
\hline 0.06 & 0.00000000 & -0.04527210 & -0.07203318 & -0.07311263 & -0.05460161 & -0.02784053 & 0.00000000 \\
\hline 0.08 & 0.00000000 & -0.04546123 & -0.07073068 & -0.06903743 & -0.04884565 & -0.02357620 & 0.00000000 \\
\hline 0.10 & 0.00000000 & -0.04561907 & -0.06954816 & -0.06537551 & -0.04368554 & -0.01975644 & 0.00000000 \\
\hline 0.14 & 0.00000000 & -0.04587994 & -0.06749869 & -0.05906351 & -0.03480231 & -0.01318357 & 0.00000000 \\
\hline 0.16 & 0.00000000 & -0.04599242 & -0.06660663 & -0.05631905 & -0.03094083 & -0.01032663 & 0.00000000 \\
\hline 0.18 & 0.00000000 & -0.04609615 & -0.06578780 & -0.05379860 & -0.02739410 & -0.00770245 & 0.00000000 \\
\hline 0.20 & 0.00000000 & -0.04619240 & -0.06503252 & -0.05147216 & -0.02411988 & -0.00527976 & 0.00000000 \\
\hline 0.22 & 0.00000000 & -0.04628197 & -0.06433238 & -0.04931465 & -0.02108310 & -0.00303268 & 0.00000000 \\
\hline 0.24 & 0.00000000 & -0.04636537 & -0.06368019 & -0.04730497 & -0.01825442 & -0.00093960 & 0.00000000 \\
\hline 0.26 & 0.00000000 & -0.04644292 & -0.06306972 & -0.04542527 & -0.01560915 & 0.00101765 & 0.00000000 \\
\hline 0.30 & 0.00000000 & -0.04658120 & -0.06195326 & -0.04199724 & -0.01078809 & 0.00458397 & 0.00000000 \\
\hline 0.32 & 0.00000000 & -0.04664212 & -0.06143858 & -0.04042469 & -0.00857903 & 0.00621743 & 0.00000000 \\
\hline 0.34 & 0.00000000 & -0.04669761 & -0.06094808 & -0.03893301 & -0.00648587 & 0.00776460 & 0.00000000 \\
\hline 0.36 & 0.00000000 & -0.04674766 & -0.06047864 & -0.03751372 & -0.00449703 & 0.00923395 & 0.00000000 \\
\hline 0.38 & 0.00000000 & -0.04679226 & -0.06002752 & -0.03615939 & -0.00260238 & 0.01063288 & 0.00000000 \\
\hline 0.40 & 0.00000000 & -0.04683137 & -0.05959228 & -0.03486346 & -0.00079301 & 0.01196790 & 0.00000000 \\
\hline 0.42 & 0.00000000 & -0.04686491 & -0.05917073 & -0.03362013 & 0.00093897 & 0.01324479 & 0.00000000 \\
\hline 0.44 & 0.00000000 & -0.04689283 & -0.05876091 & -0.03242418 & 0.00260057 & 0.01446865 & 0.00000000 \\
\hline 0.46 & 0.00000000 & -0.04691503 & -0.05836100 & -0.03127097 & 0.00419809 & 0.01564406 & 0.00000000 \\
\hline 0.48 & 0.00000000 & -0.04693140 & -0.05796936 & -0.03015627 & 0.00573717 & 0.01677513 & 0.00000000 \\
\hline 0.50 & 0.00000000 & -0.04694181 & -0.05758445 & -0.02907623 & 0.00722294 & 0.01786558 & 0.00000000 \\
\hline 0.52 & 0.00000000 & -0.04694612 & -0.05720485 & -0.02802736 & 0.00866004 & 0.01891877 & 0.00000000 \\
\hline 0.54 & 0.00000000 & -0.04694415 & -0.05682917 & -0.02700638 & 0.01005276 & 0.01993778 & 0.00000000 \\
\hline 0.56 & 0.00000000 & -0.04693570 & -0.05645612 & -0.02601026 & 0.01140502 & 0.02092544 & 0.00000000 \\
\hline 0.58 & 0.00000000 & -0.04692054 & -0.05608442 & -0.02503617 & 0.01272050 & 0.02188437 & 0.00000000 \\
\hline 0.64 & 0.00000000 & -0.04683184 & -0.05496466 & -0.02221928 & 0.01647974 & 0.02461256 & 0.00000000 \\
\hline 0.66 & 0.00000000 & -0.04678663 & -0.05458550 & -0.02130691 & 0.01768085 & 0.02547972 & 0.00000000 \\
\hline 0.68 & 0.00000000 & -0.04673282 & -0.05420105 & -0.02040360 & 0.01886098 & 0.02632921 & 0.00000000 \\
\hline 0.70 & 0.00000000 & -0.04666979 & -0.05380975 & -0.01950674 & 0.02002309 & 0.02716305 & 0.00000000 \\
\hline 0.72 & 0.00000000 & -0.04659683 & -0.05340987 & -0.01861357 & 0.02117022 & 0.02798326 & 0.00000000 \\
\hline 0.74 & 0.00000000 & -0.04651308 & -0.05299948 & -0.01772118 & 0.02230550 & 0.02879190 & 0.00000000 \\
\hline 0.76 & 0.00000000 & -0.04641747 & -0.05257633 & -0.01682632 & 0.02343229 & 0.02959115 & 0.00000000 \\
\hline 0.78 & 0.00000000 & -0.04630872 & -0.05213782 & -0.01592538 & 0.02455425 & 0.03038334 & 0.00000000 \\
\hline 0.80 & 0.00000000 & -0.04618520 & -0.05168076 & -0.01501414 & 0.02567551 & 0.03117106 & 0.00000000 \\
\hline 0.82 & 0.00000000 & -0.04604484 & -0.05120125 & -0.01408756 & 0.02680088 & 0.03195728 & 0.00000000 \\
\hline 0.84 & 0.00000000 & -0.04588496 & -0.05069432 & -0.01313943 & 0.02793616 & 0.03274553 & 0.00000000 \\
\hline 0.86 & 0.00000000 & -0.04570193 & -0.05015344 & -0.01216175 & 0.02908868 & 0.03354019 & 0.00000000 \\
\hline 0.88 & 0.00000000 & -0.04549078 & -0.04956968 & -0.01114378 & 0.03026809 & 0.03434699 & 0.00000000 \\
\hline 0.90 & 0.00000000 & -0.04524422 & -0.04893021 & -0.01007031 & 0.03148792 & 0.03517391 & 0.00000000 \\
\hline 0.92 & 0.00000000 & -0.04495100 & -0.04821528 & -0.00891818 & 0.03276854 & 0.03603282 & 0.00000000 \\
\hline 0.94 & 0.00000000 & -0.04459201 & -0.04739157 & -0.00764855 & 0.03414390 & 0.03694347 & 0.00000000 \\
\hline 0.96 & 0.00000000 & -0.04412966 & -0.04639377 & -0.00618566 & 0.03567989 & 0.03794400 & 0.00000000 \\
\hline 0.98 & 0.00000000 & -0.04346602 & -0.04505206 & -0.00433313 & 0.03754685 & 0.03913289 & 0.00000000 \\
\hline 1.00 & 0.00000000 & -0.04155586 & -0.04155586 & 0.00000000 & 0.04155586 & 0.04155586 & 0.00000000 \\
\hline
\end{tabular}

The calculation of the matrix $\mathbf{A}$ and the $M$-functions, and their relation to the so-called $H$-functions, are described in Appendices A and B, respectively. Equation (8a) can be recast in terms of the so-called H-matrix (De Rooij et al. 1989), which is also related to the $H$-functions (see Appendix C).

We have tabulated results for values of optical thickness up to 1024 and then for a semi-infinite slab. Here we provide asymptotic expressions to obtain Stokes parameters of a thick atmosphere with a black surface underneath, from those of a semi-infinite atmosphere. For further details on the derivation of these expressions, the readers are referred to Domke
(1978), van de Hulst (1980), De Rooij (1985), Wauben et al. (1994), and Hovenier et al. (2004). The formulae are based on the fact that we have accurate numbers for the Stokes parameters of light reflected by a semi-infinite atmosphere and that the azimuth dependence of the Stokes parameters of reflected light for a corresponding thick atmosphere is close to that for the semi-infinite atmosphere, while the azimuth dependence of the Stokes parameters of transmitted light can be neglected.

Suppose we know the Stokes parameters for diffuse reflection emerging from the top of a semi-infinite atmosphere. Compute 
Table 6

$U$ (Transmission at BOA) for $b=16, \mu_{0}=0.2$, and $A=0$

\begin{tabular}{|c|c|c|c|c|c|c|c|}
\hline$\mu$ & $\varphi=0^{\circ}$ & $\varphi=30^{\circ}$ & $\varphi=60^{\circ}$ & $\varphi=90^{\circ}$ & $\varphi=120^{\circ}$ & $\varphi=150^{\circ}$ & $\varphi=180^{\circ}$ \\
\hline 0.00 & 0.00000000 & 0.00000000 & 0.00000000 & 0.00000000 & 0.00000000 & 0.00000000 & 0.00000000 \\
\hline 0.02 & 0.00000000 & 0.00000000 & 0.00000000 & 0.00000000 & 0.00000000 & 0.00000000 & 0.00000000 \\
\hline 0.04 & 0.00000000 & 0.00000000 & 0.00000000 & 0.00000000 & -0.00000001 & -0.00000001 & 0.00000000 \\
\hline 0.06 & 0.00000000 & 0.00000001 & 0.00000000 & 0.00000000 & -0.00000001 & -0.00000001 & 0.00000000 \\
\hline 0.08 & 0.00000000 & 0.00000001 & 0.00000001 & 0.00000000 & -0.00000001 & -0.00000001 & 0.00000000 \\
\hline 0.10 & 0.00000000 & 0.00000001 & 0.00000001 & 0.00000000 & -0.00000001 & -0.00000001 & 0.00000000 \\
\hline 0.14 & 0.00000000 & 0.00000002 & 0.00000001 & 0.00000000 & -0.00000002 & -0.00000002 & 0.00000000 \\
\hline 0.16 & 0.00000000 & 0.00000002 & 0.00000002 & 0.00000000 & -0.00000002 & -0.00000002 & 0.00000000 \\
\hline 0.18 & 0.00000000 & 0.00000002 & 0.00000002 & 0.00000000 & -0.00000003 & -0.00000003 & 0.00000000 \\
\hline 0.20 & 0.00000000 & 0.00000003 & 0.00000002 & 0.00000000 & -0.00000003 & -0.00000003 & 0.00000000 \\
\hline 0.22 & 0.00000000 & 0.00000003 & 0.00000003 & 0.00000000 & -0.00000003 & -0.00000003 & 0.00000000 \\
\hline 0.24 & 0.00000000 & 0.00000003 & 0.00000003 & 0.00000000 & -0.00000004 & -0.00000004 & 0.00000000 \\
\hline 0.26 & 0.00000000 & 0.00000004 & 0.00000003 & 0.00000000 & -0.00000004 & -0.00000004 & 0.00000000 \\
\hline 0.30 & 0.00000000 & 0.00000004 & 0.00000004 & 0.00000000 & -0.00000005 & -0.00000005 & 0.00000000 \\
\hline 0.32 & 0.00000000 & 0.00000005 & 0.00000005 & 0.00000000 & -0.00000006 & -0.00000005 & 0.00000000 \\
\hline 0.34 & 0.00000000 & 0.00000005 & 0.00000005 & -0.00000001 & -0.00000006 & -0.00000006 & 0.00000000 \\
\hline 0.36 & 0.00000000 & 0.00000006 & 0.00000006 & -0.00000001 & -0.00000006 & -0.00000006 & 0.00000000 \\
\hline 0.38 & 0.00000000 & 0.00000006 & 0.00000006 & -0.00000001 & -0.00000007 & -0.00000007 & 0.00000000 \\
\hline 0.40 & 0.00000000 & 0.00000007 & 0.00000007 & -0.00000001 & -0.00000008 & -0.00000007 & 0.00000000 \\
\hline 0.42 & 0.00000000 & 0.00000007 & 0.00000007 & -0.00000001 & -0.00000008 & -0.00000008 & 0.00000000 \\
\hline 0.44 & 0.00000000 & 0.00000008 & 0.00000008 & -0.00000001 & -0.00000009 & -0.00000009 & 0.00000000 \\
\hline 0.46 & 0.00000000 & 0.00000009 & 0.00000008 & -0.00000001 & -0.00000009 & -0.00000009 & 0.00000000 \\
\hline 0.48 & 0.00000000 & 0.00000009 & 0.00000009 & -0.00000001 & -0.00000010 & -0.00000010 & 0.00000000 \\
\hline 0.50 & 0.00000000 & 0.00000010 & 0.00000010 & -0.00000001 & -0.00000011 & -0.00000010 & 0.00000000 \\
\hline 0.52 & 0.00000000 & 0.00000011 & 0.00000010 & -0.00000001 & -0.00000011 & -0.00000011 & 0.00000000 \\
\hline 0.54 & 0.00000000 & 0.00000011 & 0.00000011 & -0.00000001 & -0.00000012 & -0.00000012 & 0.00000000 \\
\hline 0.56 & 0.00000000 & 0.00000012 & 0.00000012 & -0.00000001 & -0.00000013 & -0.00000013 & 0.00000000 \\
\hline 0.58 & 0.00000000 & 0.00000013 & 0.00000013 & -0.00000001 & -0.00000014 & -0.00000014 & 0.00000000 \\
\hline 0.64 & 0.00000000 & 0.00000016 & 0.00000015 & -0.00000001 & -0.00000017 & -0.00000016 & 0.00000000 \\
\hline 0.66 & 0.00000000 & 0.00000017 & 0.00000016 & -0.00000001 & -0.00000018 & -0.00000018 & 0.00000000 \\
\hline 0.68 & 0.00000000 & 0.00000018 & 0.00000018 & -0.00000001 & -0.00000019 & -0.00000019 & 0.00000000 \\
\hline 0.70 & 0.00000000 & 0.00000019 & 0.00000019 & -0.00000001 & -0.00000020 & -0.00000020 & 0.00000000 \\
\hline 0.72 & 0.00000000 & 0.00000020 & 0.00000020 & -0.00000001 & -0.00000021 & -0.00000021 & 0.00000000 \\
\hline 0.74 & 0.00000000 & 0.00000022 & 0.00000021 & -0.00000001 & -0.00000023 & -0.00000023 & 0.00000000 \\
\hline 0.76 & 0.00000000 & 0.00000023 & 0.00000023 & -0.00000001 & -0.00000024 & -0.00000024 & 0.00000000 \\
\hline 0.78 & 0.00000000 & 0.00000025 & 0.00000024 & -0.00000001 & -0.00000026 & -0.00000026 & 0.00000000 \\
\hline 0.80 & 0.00000000 & 0.00000026 & 0.00000026 & -0.00000001 & -0.00000028 & -0.00000027 & 0.00000000 \\
\hline 0.82 & 0.00000000 & 0.00000028 & 0.00000028 & -0.00000001 & -0.00000029 & -0.00000029 & 0.00000000 \\
\hline 0.84 & 0.00000000 & 0.00000030 & 0.00000030 & -0.00000001 & -0.00000031 & -0.00000031 & 0.00000000 \\
\hline 0.86 & 0.00000000 & 0.00000032 & 0.00000032 & -0.00000001 & -0.00000034 & -0.00000033 & 0.00000000 \\
\hline 0.88 & 0.00000000 & 0.00000034 & 0.00000034 & -0.00000001 & -0.00000036 & -0.00000036 & 0.00000000 \\
\hline 0.90 & 0.00000000 & 0.00000037 & 0.00000037 & -0.00000001 & -0.00000038 & -0.00000038 & 0.00000000 \\
\hline 0.92 & 0.00000000 & 0.00000040 & 0.00000039 & -0.00000001 & -0.00000041 & -0.00000041 & 0.00000000 \\
\hline 0.94 & 0.00000000 & 0.00000043 & 0.00000042 & -0.00000001 & -0.00000044 & -0.00000044 & 0.00000000 \\
\hline 0.96 & 0.00000000 & 0.00000046 & 0.00000046 & -0.00000001 & -0.00000047 & -0.00000047 & 0.00000000 \\
\hline 0.98 & 0.00000000 & 0.00000050 & 0.00000049 & -0.00000001 & -0.00000051 & -0.00000050 & 0.00000000 \\
\hline 1.00 & 0.00000000 & 0.00000054 & 0.00000054 & 0.00000000 & -0.00000054 & -0.00000054 & 0.00000000 \\
\hline
\end{tabular}

$K_{11}(\mu)$ and $K_{21}(\mu)$ as follows:

$$
\begin{array}{r}
K_{11}(\mu)=\frac{3}{4}\left[\mu+2 \int_{0}^{1} \frac{\mu^{\prime}}{F_{0}} I_{\infty}^{0}\left(0 ; \mu, \mu^{\prime}\right) d \mu^{\prime}\right] ; \\
K_{21}(\mu)=\frac{3}{2} \int_{0}^{1} \frac{\mu^{\prime}}{F_{0}} Q_{\infty}^{0}\left(0 ; \mu, \mu^{\prime}\right) d \mu^{\prime} .
\end{array}
$$

The subscript $\infty$ implies that the optical thickness of the atmosphere is infinity.
Equation (10a) can be used to calculate $q_{0}$ :

$$
q_{0}=2 \int_{0}^{1} \mu^{2} K_{11}(\mu) d \mu
$$

For large optical thickness $b$, we have the following asymptotic expressions:

$$
I(0 ; \mu, \varphi)=I_{\infty}(0 ; \mu, \varphi)-\frac{4 \mu_{0} F_{0} K_{11}\left(\mu_{0}\right) K_{11}(\mu)}{3\left(b+2 q_{0}\right)} ;
$$


Table 7

$I$ (Reflection at TOA) for $\mu_{0}=0.2, \mu=0.4, \varphi=60^{\circ}$, and $A=0.25$

\begin{tabular}{lcccc}
\hline \hline & $b=2$ & $b=16$ & $b=100$ & $b=\infty$ \\
\hline This work & 0.17966823 & 0.20618483 & 0.21211230 & 0.21337320 \\
Sekera/Kahle & 0.17963857 & 0.20615855 & 0.21208537 & $\ldots$ \\
\hline
\end{tabular}

Table 8

$Q$ (Reflection at TOA) for $\mu_{0}=0.2, \mu=0.4, \varphi=60^{\circ}$, and $A=0.25$

\begin{tabular}{lcccc}
\hline \hline & $b=2$ & $b=16$ & $b=100$ & $b=\infty$ \\
\hline This work & 0.07073418 & 0.07034871 & 0.07016895 & 0.07013071 \\
Sekera/Kahle & 0.07071139 & 0.07032380 & 0.07014505 & $\ldots$ \\
\hline
\end{tabular}

Table 9

$U$ (Reflection at TOA) for $\mu_{0}=0.2, \mu=0.4, \varphi=60^{\circ}$, and $A=0.25$

\begin{tabular}{lcccc}
\hline \hline & $b=2$ & $b=16$ & $b=100$ & $b=\infty$ \\
\hline This work & -0.05937699 & -0.05959228 & -0.05959228 & -0.05959228 \\
Sekera/Kahle & -0.05937691 & -0.05959003 & -0.05959003 & $\ldots$ \\
\hline
\end{tabular}

Table 10

$I$ (Transmission at BOA) for $\mu_{0}=0.2, \mu=0.4, \varphi=60^{\circ}$, and $A=0.25$

\begin{tabular}{lccc}
\hline \hline & $b=2$ & $b=16$ & $b=100$ \\
\hline This work & 0.05205555 & 0.01024465 & 0.00179701 \\
Sekera/Kahle & 0.05206533 & 0.01024642 & 0.00179730 \\
\hline
\end{tabular}

Table 11

$Q$ (Transmission at BOA) for $\mu_{0}=0.2, \mu=0.4, \varphi=60^{\circ}$, and $A=0.25$

\begin{tabular}{lccc}
\hline \hline & $b=2$ & $b=16$ & $b=100$ \\
\hline This work & 0.00787490 & -0.00021793 & -0.00003824 \\
Sekera/Kahle & 0.00787632 & -0.00021743 & -0.00003810 \\
\hline
\end{tabular}

Table 12

$U$ (Transmission at BOA) for $\mu_{0}=0.2, \mu=0.4, \varphi=60^{\circ}$, and $A=0.25$

\begin{tabular}{lccc}
\hline \hline & $b=2$ & $b=16$ & $b=100$ \\
\hline This work & 0.00021790 & 0.00000007 & 0.00000000 \\
Sekera/Kahle & 0.00021732 & 0.00000007 & 0.00000000 \\
\hline
\end{tabular}

$$
\begin{gathered}
Q(0 ; \mu, \varphi)=Q_{\infty}(0 ; \mu, \varphi)-\frac{4 \mu_{0} F_{0} K_{11}\left(\mu_{0}\right) K_{21}(\mu)}{3\left(b+2 q_{0}\right)} . \\
I(b ; \mu, \varphi)=\frac{4 \mu_{0} F_{0} K_{11}\left(\mu_{0}\right) K_{11}(\mu)}{3\left(b+2 q_{0}\right)} ; \\
Q(b ; \mu, \varphi)=\frac{4 \mu_{0} F_{0} K_{11}\left(\mu_{0}\right) K_{21}(\mu)}{3\left(b+2 q_{0}\right)} .
\end{gathered}
$$

Furthermore, if we know Stokes parameter $U_{\infty}$ for diffuse reflection emerging from the top of a semi-infinite atmosphere, we have the relations:

$$
\begin{gathered}
U(0 ; \mu, \varphi)=U_{\infty}(0 ; \mu, \varphi) ; \\
U(b ; \mu, \varphi)=0 .
\end{gathered}
$$

\section{RESULTS AND DISCUSSION}

The main purpose of this paper is to provide benchmark results for Stokes parameters $I, Q$, and $U$, for reflection at the top
Table 13

Stokes parameters (Reflection at TOA) for $b=1024, \mu_{0}=0.2$, $\mu=0.4, \varphi=60^{\circ}$, and $A=0$

\begin{tabular}{lccc}
\hline \hline & $I$ & $Q$ & $U$ \\
\hline Exact computation & 0.21324794 & 0.07013451 & -0.05959228 \\
Asymptotic computation & 0.21324795 & 0.07013451 & -0.05959228 \\
\hline
\end{tabular}

Table 14

Stokes parameters (Transmission at BOA) for $b=1024, \mu_{0}=0.2$, $\mu=0.4, \varphi=60^{\circ}$, and $A=0$

\begin{tabular}{lccc}
\hline \hline & $I$ & $Q$ & $U$ \\
\hline Exact computation & 0.00012526 & -0.00000380 & 0.00000000 \\
Asymptotic computation & 0.00012525 & -0.00000380 & 0.00000000 \\
\hline
\end{tabular}

Table 15

Stokes Parameters (Reflection at TOA) for $b=32, \mu_{0}=0.2$, $\mu=0.4, \varphi=60^{\circ}$, and $A=0$

\begin{tabular}{lccc}
\hline \hline & $I$ & $Q$ & $U$ \\
\hline Exact computation & 0.20953028 & 0.07024725 & -0.05959228 \\
Asymptotic computation & 0.20953028 & 0.07024725 & -0.05959228 \\
\hline
\end{tabular}

Table 16

Stokes Parameters (Transmission at BOA) for $b=32, \mu_{0}=0.2$, $\mu=0.4, \varphi=60^{\circ}$, and $A=0$

\begin{tabular}{lccc}
\hline \hline & $I$ & $Q$ & $U$ \\
\hline Exact computation & 0.00384292 & -0.00011654 & 0.00000000 \\
Asymptotic computation & 0.00384292 & -0.00011654 & 0.00000000 \\
\hline
\end{tabular}

and transmission at the bottom, of thick non-absorbing homogeneous Rayleigh scattering atmospheres, illuminated at the top by a parallel beam of unpolarized light with net flux $\pi$ per unit area perpendicular to the incident beam, and bounded below by a Lambert surface with albedo $A$. To this end, we have tabulated results for 50 values of $\mu_{0}(0.02-1$ in steps of 0.02$), 51$ values of $\mu(0-1$ in steps of 0.02$), 7$ values of $\varphi\left(0^{\circ}-180^{\circ}\right.$ in steps of $\left.30^{\circ}\right)$, 3 values of $A(0,0.25,0.8)$, and 13 values of $b(1,2,4,8,16$, $32,64,100,128,256,512,1024, \infty)$. These results can be obtained from the following Web site: http://resolver.caltech.edu/ CaltechAUTHORS:20111110-130941966. The results have been tabulated to eight decimal places; this is an indication of the precision of our results and is discussed in detail in the next section. Note that due to symmetry with respect to the principal plane, which is defined by $\varphi=0$ and $\varphi=\pi, I$ and $Q$ have the same values for $2 \pi-\varphi$ as they have for $\varphi$, but $U$ has the opposite sign for those two azimuthal directions (cf. Equations 7(a)-7(c)). Therefore, we have restricted the tables to $\varphi$ in the range $[0, \pi]$. Here we present sample results for $I$ (Tables 1 and 2), $Q$ (Tables 3 and 4), and $U$ (Tables 5 and 6). In Tables $1-6, b=16, \mu_{0}=0.2$, and $A=0$.

Sekera \& Kahle (1966) generated tables of the $X$ - and $Y$-functions and the related $K$ - and $L$-functions for a range of optical thickness values between 0.15 and 100 . They also generated tables of the moments of these functions. These are the only tables available for the values of $b$ we are interested in. Hence, we tabulate improved results for these functions on the following Web site: http://resolver.caltech.edu/ CaltechAUTHORS:20120206-140559033. Note that the ordered pairs of functions $\left(X_{1}, Y_{1}\right),\left(X_{2}, Y_{2}\right),\left(X_{3}, Y_{3}\right)$ and $\left(X_{4}, Y_{4}\right)$ used by Sekera \& Kahle (1966) are equivalent, respectively, to the functions $\left(X^{1}, Y^{1}\right),\left(X^{2}, Y^{2}\right),\left(X_{l}, Y_{l}\right)$ and $\left(X_{r}, Y_{r}\right)$ of Chandrasekhar \& Elbert (1954). Furthermore, the characteristic 

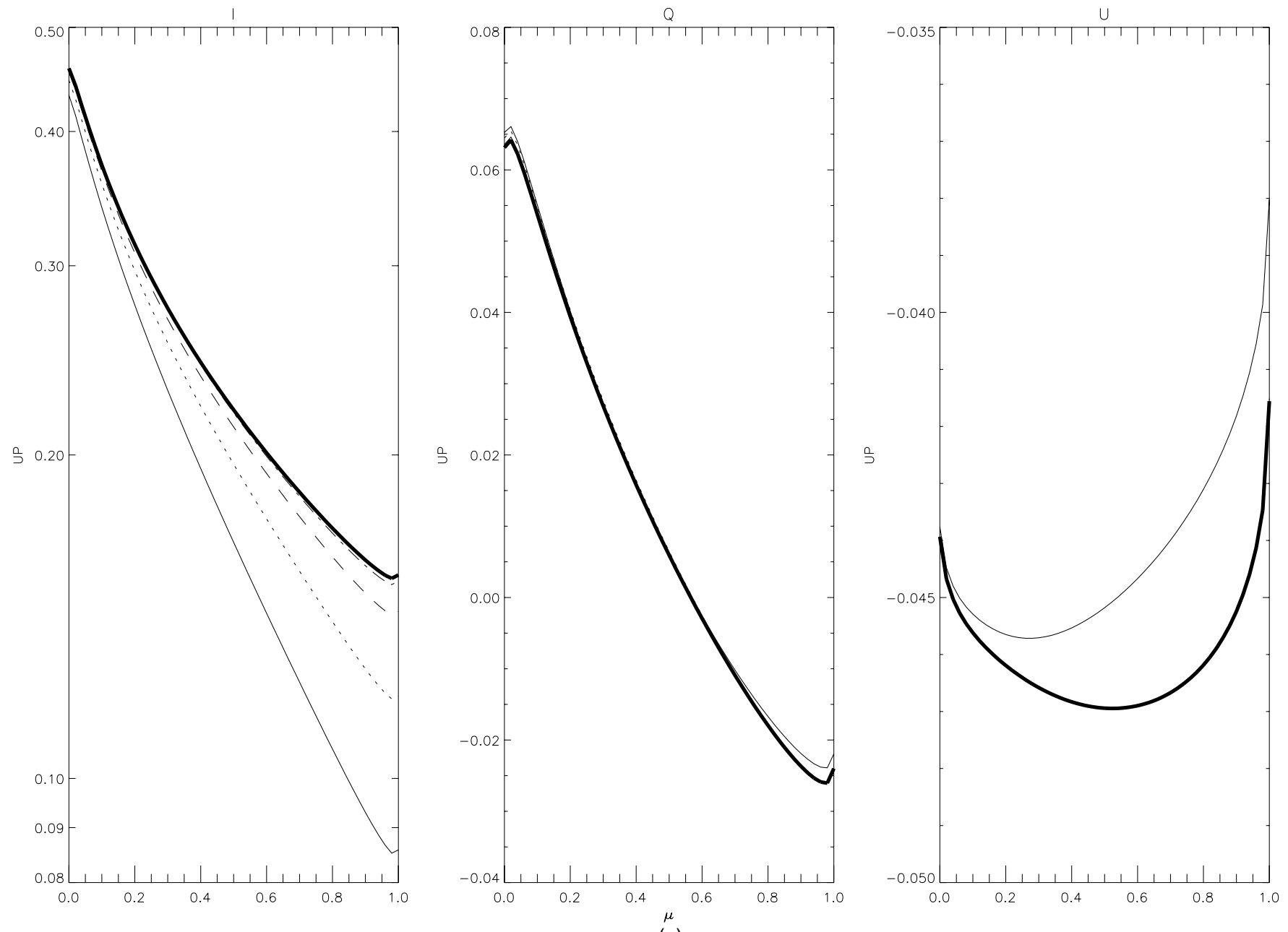

(a)

Figure 2. Stokes parameters as functions of $\mu$ for (a) reflection at the top of the atmosphere and (b) transmission at the bottom of the atmosphere; (c) degree and direction of linear polarization as functions of $\mu . \mu_{0}=0.2, \varphi=30^{\circ}, A=0.25, b=1$ (solid), 4 (dotted), 16 (dashed), 100 (dash-dot), and (for reflection only) $\infty$ (solid bold).

function $\Psi_{l}$ is conservative, and $\left(X_{l}, Y_{l}\right)$ do not have a unique solution (Chandrasekhar 1950). We use the standard solution of Chandrasekhar (1950), whereas Sekera \& Kahle (1966) use the solution of Busbridge (1960) with analytical behavior for $\mu \rightarrow \infty$.

Tables 7-9 compare the Stokes parameters for reflection at the TOA obtained by us with those from Sekera \& Kahle (1966) for three values of the optical thickness $(2,16,100)$ and an albedo $A=0.25$ for a Lambert surface below the atmosphere. We also list our values for a semi-infinite atmosphere. Tables 10-12 do the same for transmission at the BOA. Note that Sekera \& Kahle (1966) did not tabulate Stokes parameters; we used their formulae and tables of auxiliary functions to compute the Stokes parameters in Tables 7-12, using our definitions of Stokes parameters and directional parameters (see Section 2). Also, it is not possible to obtain Stokes parameters from their tabulated functions for the case of transmission in the direction of the incoming light, due to the singularity in the calculation of the transmission functions. This special case would require knowledge of the derivatives of the $X$ and $Y$ functions, which they do not provide.

It is clear that Stokes parameters $I$ and $Q$ for reflection at $b=$ 100 have not reached the values (to eight decimal places) for semi-infinite atmospheres. Sekera \& Kahle (1966) further use several relations to illustrate the accuracy of their computations. However, many of their tests say very little about the reliability of their results for the Stokes parameters. Our results satisfy all of their relations to eight decimal places; yet, the values of our Stokes parameters differ from theirs to the same precision. In the next section, we describe the tests performed to validate the precision of our results.

Figures 2(a) and (b) illustrate how the Stokes parameters, respectively, for reflection at the TOA, and transmission at the BOA, vary with $\mu$ for several values of optical thickness. It is apparent that Stokes parameters $Q$ and $U$ for reflection converge to the value for semi-infinite atmospheres much quicker than does Stokes parameter I. Figure 2(c) illustrates how the degree and direction of linear polarization vary for the same parameters.

We show the utility of the asymptotic expressions in Tables 13-16. It is immediately evident that these formulae are a very useful tool to obtain Stokes parameters for optically thick atmospheres from the values for the corresponding semiinfinite atmosphere. In fact, they are good enough to provide results correct to within one unit of the eighth decimal place for values of $b$ as low as 32. They can also be used for interpolation between two values of the optical thickness for which tables are presented. 

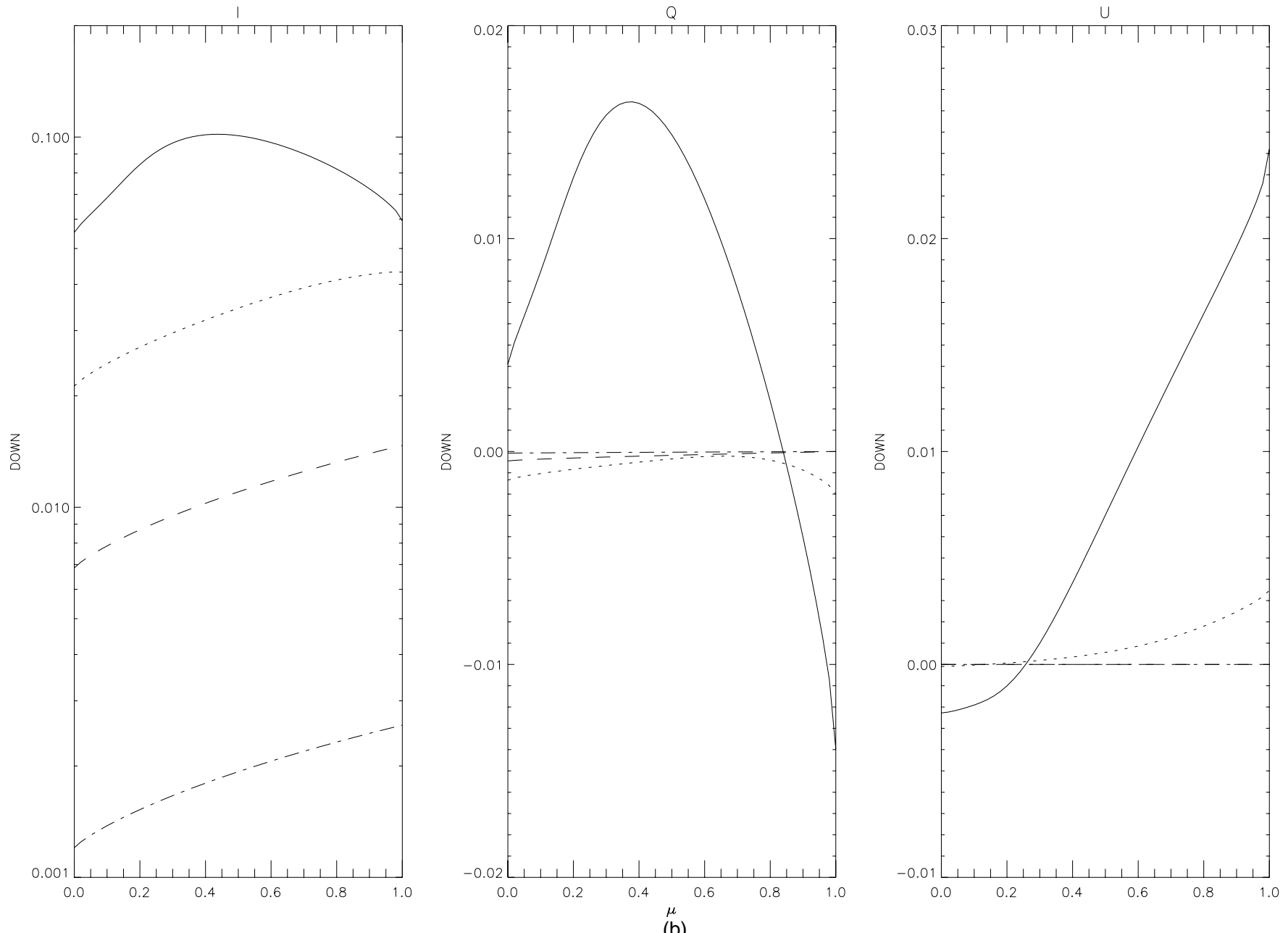

(b)
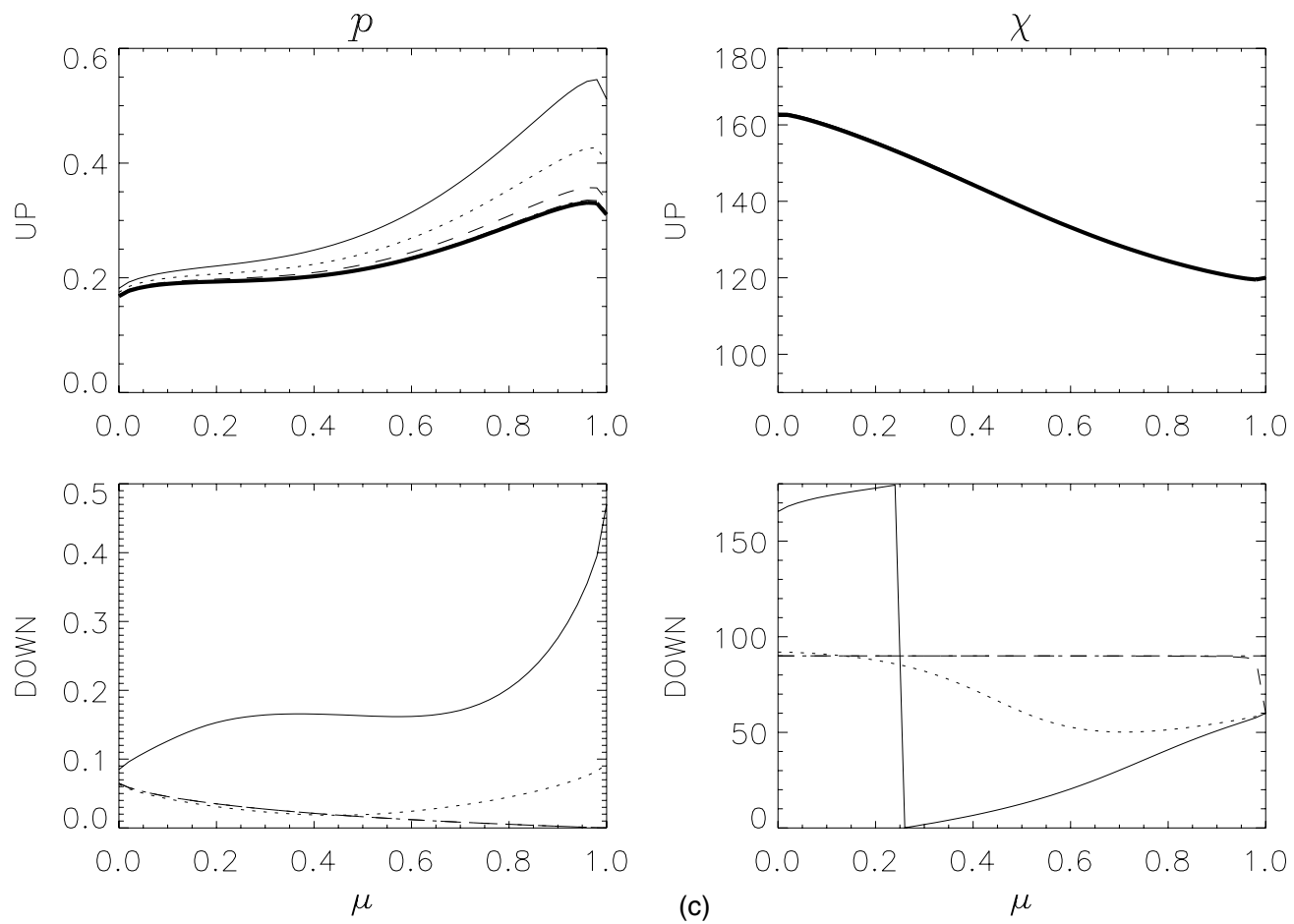

Figure 2. (Continued) 

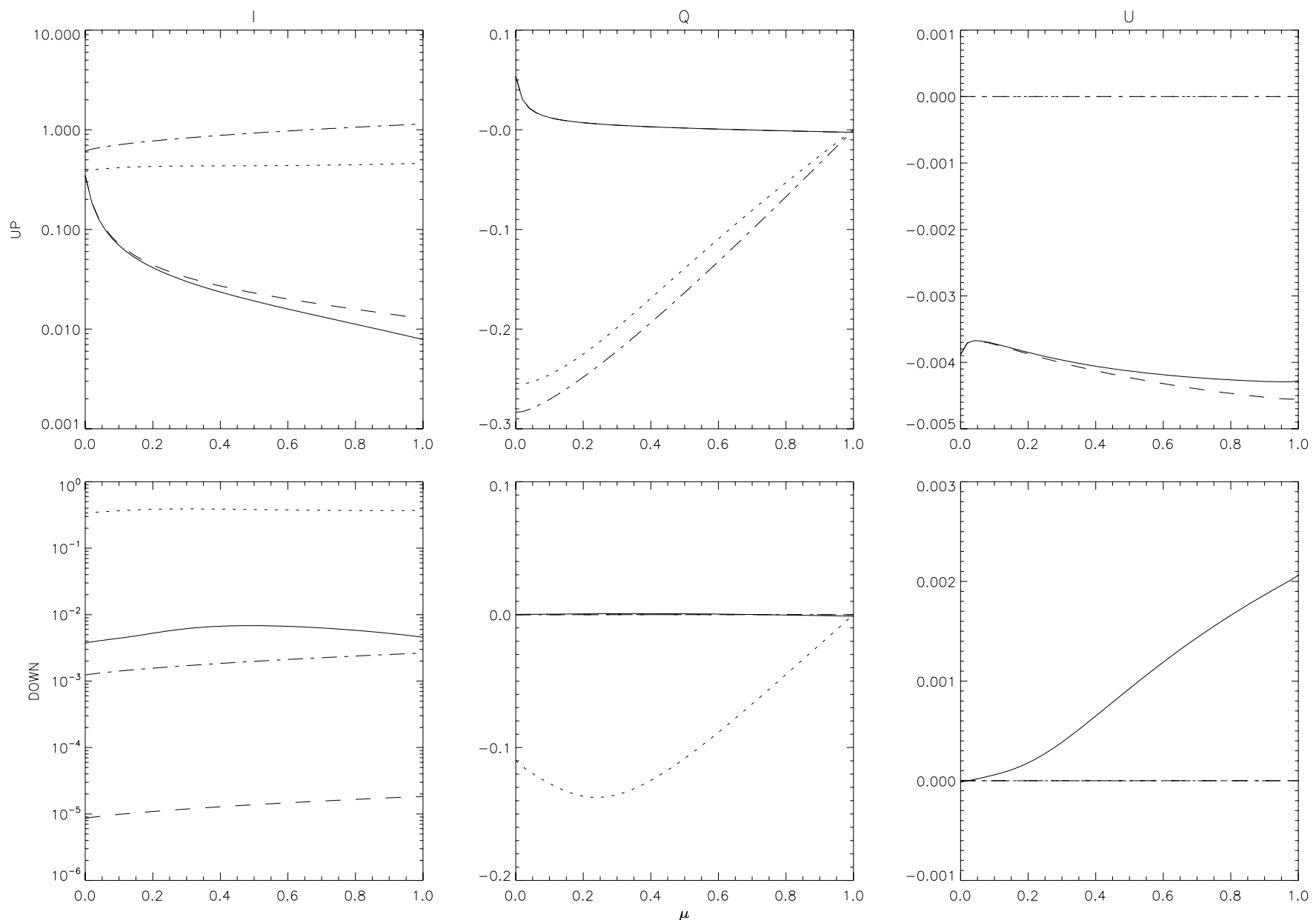

(a)

Figure 3. (a) Stokes parameters as functions of $\mu$ for $\varphi=30^{\circ}$ and $A=0.25$. $\left(b, \mu_{0}\right)=(1,0.02)$ (solid), $(1,1.0)$ (dotted), (1024, 0.02) (dashed), and (1024, 1.0) (dash-dotted); (b) difference (multiplied by $10^{8}$ ) of doubling-adding results from the present work. The differences are positive if the doubling-adding results are larger than our results, and negative if the reverse is true. Note that the differences take only the discrete integer values $-1,0$ and 1 .

\section{VALIDATION OF RESULTS}

All results on the Web site http://resolver.caltech.edu/ CaltechAUTHORS:20111110-130941966 and in this paper have been tabulated to eight decimal places. We expect that our results are accurate to within one unit of the last decimal place. We confirmed the precision of our results by performing the following basic tests on the entire set:

1. increasing the number of quadrature points;

2. increasing the number of integration subintervals;

3. using a different numerical integration technique;

4. using quadruple precision arithmetic; and

5. comparing with results from the Doubling-Adding KNMI (DAK) code (de Haan et al. 1987) and the discrete ordinates code VLIDORT (Spurr 2006).

We obtained excellent agreement with results from the DAK code. To understand the differences between our results and the DAK results, we plot the Stokes parameters as functions of $\mu$ (Figure 3) and $b$ (Figure 4). In each of these figures, the values of the parameters (as per our computations) are first plotted, followed by the residuals between the DAK results and our results. The residuals have been multiplied by $10^{8}$ to show the difference in the eighth decimal place. Furthermore, the residuals are positive if the DAK results are larger than our results, and negative if the DAK results are smaller than our results. For example, a residual of 5 implies that the DAK results are larger than ours by five units in the eighth decimal place. Two things are evident. First, the maximum difference is one unit in the eighth decimal place. Second, the results for Stokes parameter $U$ agree perfectly between the two codes. This indicates that the difference comes from the computation of the zeroth Fourier component (which does not contribute to $U$ ).

For test 5 , it is worth noting that the agreement with the results from VLIDORT is in some cases not as good as the precision we claim. However, the larger differences are due to the fact that conservative scattering needs to be treated differently from non-conservative scattering if the discrete ordinate method is used (Siewert 2000). VLIDORT approximates conservative scattering by using a single scattering albedo slightly less than one (as specified by a user tolerance).

Nineteen different quintets $\left(\mu, \mu_{0}, \varphi, b, A\right)$ were then chosen for the reflected as well as the transmitted light to perform the following additional tests:

1. Reciprocity requires Equation (14) to hold both for reflected and transmitted light if the ground albedo is zero

$$
\mu I\left(\mu, \mu_{0}, \varphi\right)=\mu_{0} I\left(\mu_{0}, \mu, \varphi\right)
$$

If the ground albedo is not zero this relation still holds for the reflected light, but in general, not for the transmitted 

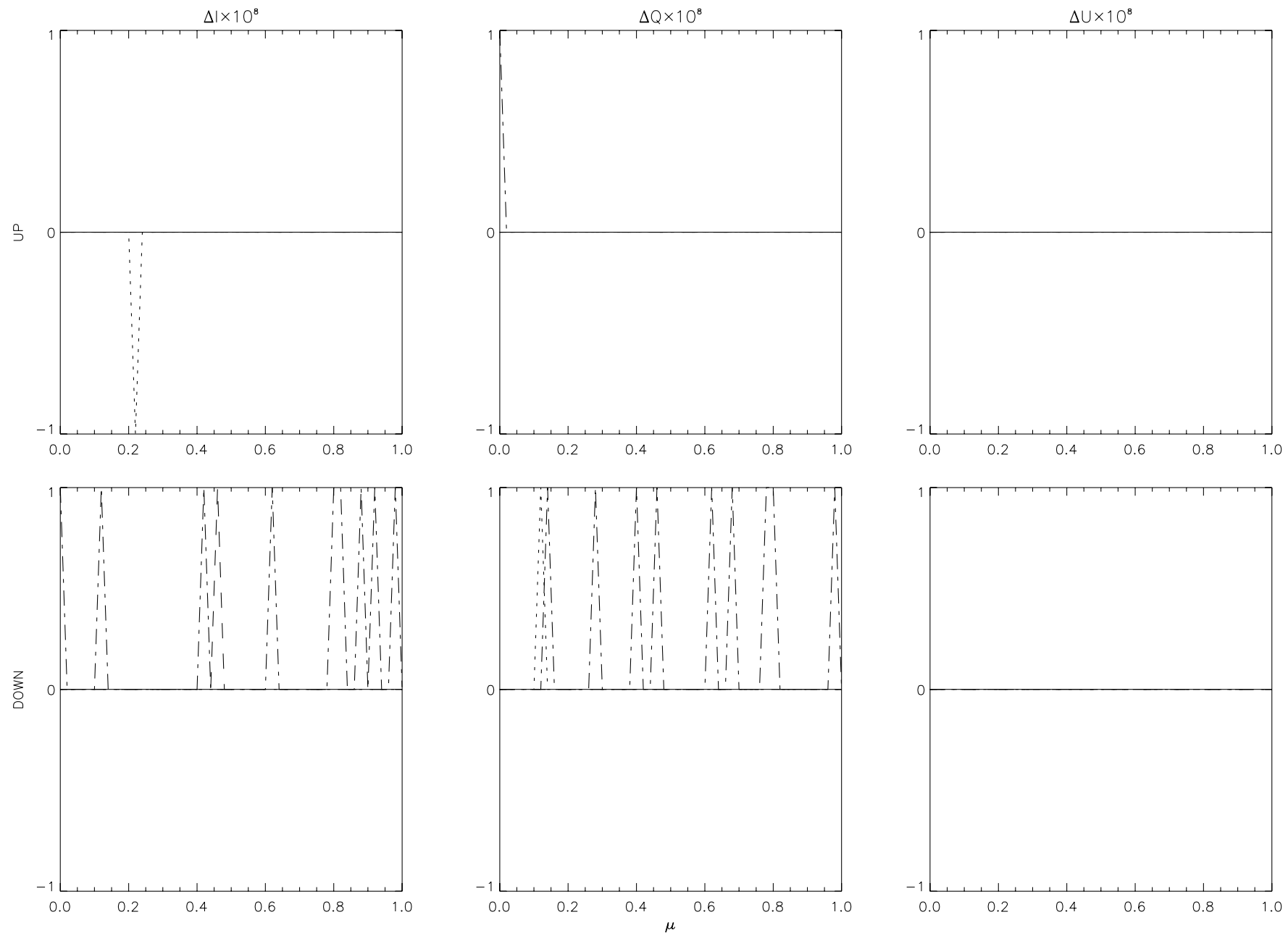

(b)

Figure 3. (Continued)

light (Hovenier 1970). The maximum difference between the left-hand side (LHS) and right-hand side (RHS) of Equation (14) for reflection is less than $10^{-12}$; the same is the case for transmission (for zero ground albedo).

2. When the incident or emerging light travels in a perpendicular direction, the azimuth dependence of the Stokes parameters of the emerging light is rather simple, as shown by Hovenier \& De Haan (1985). We found excellent agreement with the relevant formulae given by these authors.

3. When $\mu$ and $\mu_{0}$ both tend to zero the degrees of polarization $p$ and $p_{s}$ of the reflected light should both tend to their values for single scattering, according to Hovenier \& Stam (2007). By choosing $\mu=0$ and letting $\mu_{0}$ approach zero we found this indeed to be correct.

Our $H^{1}$ and $H^{2}$ functions agree with those of De Rooij et al. (1989) to 10 decimal places. Furthermore, the LHS and RHS of Equation (C10) agree to within one unit of the tenth decimal place.

For semi-infinite atmospheres, we employed the following additional relations to check the veracity of the results:

$$
\begin{gathered}
r\left(\mu_{0}\right)=2 \int_{0}^{1} \mu \frac{I_{\infty}^{0}\left(0 ; \mu, \mu_{0}\right)}{\mu_{0} F_{0}} d \mu=1 ; \\
r=2 \int_{0}^{1} \mu_{0} r\left(\mu_{0}\right) d \mu_{0}=1 .
\end{gathered}
$$

Equations (15a) and (15b) imply, respectively, that the plane albedo and the Bond albedo are unity for a semi-infinite atmosphere with conservative Rayleigh scattering. This is because, in this scenario, all incident light is ultimately reflected. These relations provide an extra check that no spurious absorption (energy loss) occurred in the computations. The plane albedo (for all values of $\mu_{0}$ ) and the Bond albedo for a semi-infinite atmosphere were found to differ from unity by less than $10^{-11}$.

\section{CONCLUSIONS}

We have computed the intensity and polarization of reflected and transmitted light in optically thick Rayleigh scattering atmospheres. Furthermore, we have provided results for semiinfinite atmospheres and given asymptotic expressions to obtain Stokes parameters for thick layers. For the sake of clarity, we have given clear definitions of Stokes parameters and related quantities such as the degree and direction of polarization. Our benchmark results have been validated using a variety of methods and are accurate to within one unit in the eighth decimal place. They can be used in particular for (exo)planetary atmospheres having a thick molecular layer above a cloud deck or solid surface, assuming the atmosphere to be locally plane-parallel. For comparisons with observations of brightness and polarization of exoplanets our benchmark results must be integrated over the illuminated part of the disk (see, e.g., Hansen \& Hovenier 1974; Stam et al. 2006). 

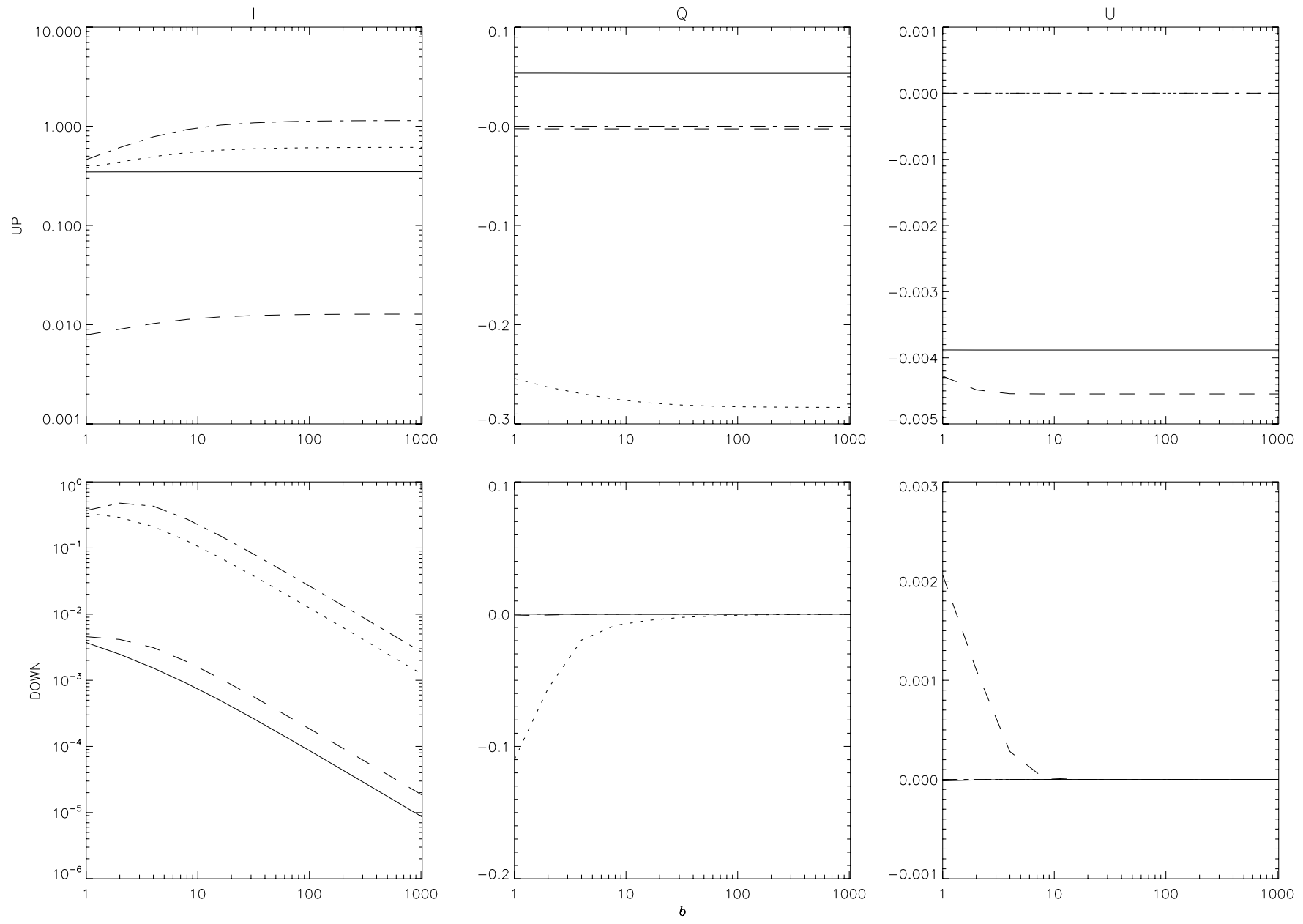

(a)

Figure 4. (a) Stokes parameters as functions of $b$ for $\varphi=30^{\circ}$ and $A=0.25 .\left(\mu, \mu_{0}\right)=(0.0,0.02)$ (solid), (0.0, 1.0) (dotted), (1.0, 0.02) (dashed), and (1.0, 1.0) (dash-dotted); (b) The difference (multiplied by $10^{8}$ ) of doubling-adding results from the present work. The differences are positive if the doubling-adding results are larger than our results and negative if the reverse is true. Note that the differences take only the discrete integer values $-1,0$, and 1 .

Part of the research described in this paper was carried out at the Jet Propulsion Laboratory at the California Institute of Technology, under a contract with the National Aeronautics and Space Administration and funded through the internal Research and Technology Development program. The authors thank Dr. Wim de Rooij for providing his code to compute the $\mathbf{H}$-matrix; Dr. Johan de Haan for the DAK code; Dr. Robert Spurr for the VLIDORT code; Prof. Yuk Yung, Ms. Irma Black, Mr. George Porter and Ms. Kathy Johnson for providing access to the two Web sites for hosting the Stokes parameter and basic function results; and the reviewer for helpful comments.

\section{APPENDIX A}

\section{THE MATRIX A EXPRESSED IN TERMS OF $H$-FUNCTIONS}

The matrix $\mathbf{A}$, and its transpose $\mathbf{A}^{\mathrm{T}}$, are related to the $H$-functions, which satisfy the nonlinear integral equation:

$$
H(\mu)=1+\mu H(\mu) \int_{0}^{1} \Psi\left(\mu^{\prime}\right)\left[\frac{H\left(\mu^{\prime}\right)}{\mu+\mu^{\prime}}\right] d \mu^{\prime} .
$$

Here $\Psi$ is called the characteristic function. For the azimuthindependent Fourier terms we require the functions $H_{l}$ and $H_{r}$ that have the characteristic functions $\Psi_{l}$ and $\Psi_{r}$, respectively:

$$
\begin{aligned}
& \Psi_{l}(\mu)=\frac{3}{4}\left(1-\mu^{2}\right) \\
& \Psi_{r}(\mu)=\frac{3}{8}\left(1-\mu^{2}\right) .
\end{aligned}
$$

We can now write:

$$
\mathbf{A}(\mu)=\left(\begin{array}{ll}
\psi(\mu) & \sqrt{2} \phi(\mu) \\
\chi(\mu) & \sqrt{2} \zeta(\mu)
\end{array}\right),
$$

where the functions $\psi$ (to be distinguished from the characteristic function $\Psi), \phi, \chi$, and $\zeta$ are related to the functions $H_{l}$ and $H_{r}$ :

$$
\begin{gathered}
\psi(\mu)=q \mu H_{l}(\mu) ; \\
\phi(\mu)=(1-c \mu) H_{l}(\mu) ; \\
\chi(\mu)=(1+c \mu) H_{r}(\mu) ; \\
\zeta(\mu)=\frac{1}{2} q \mu H_{r}(\mu) .
\end{gathered}
$$



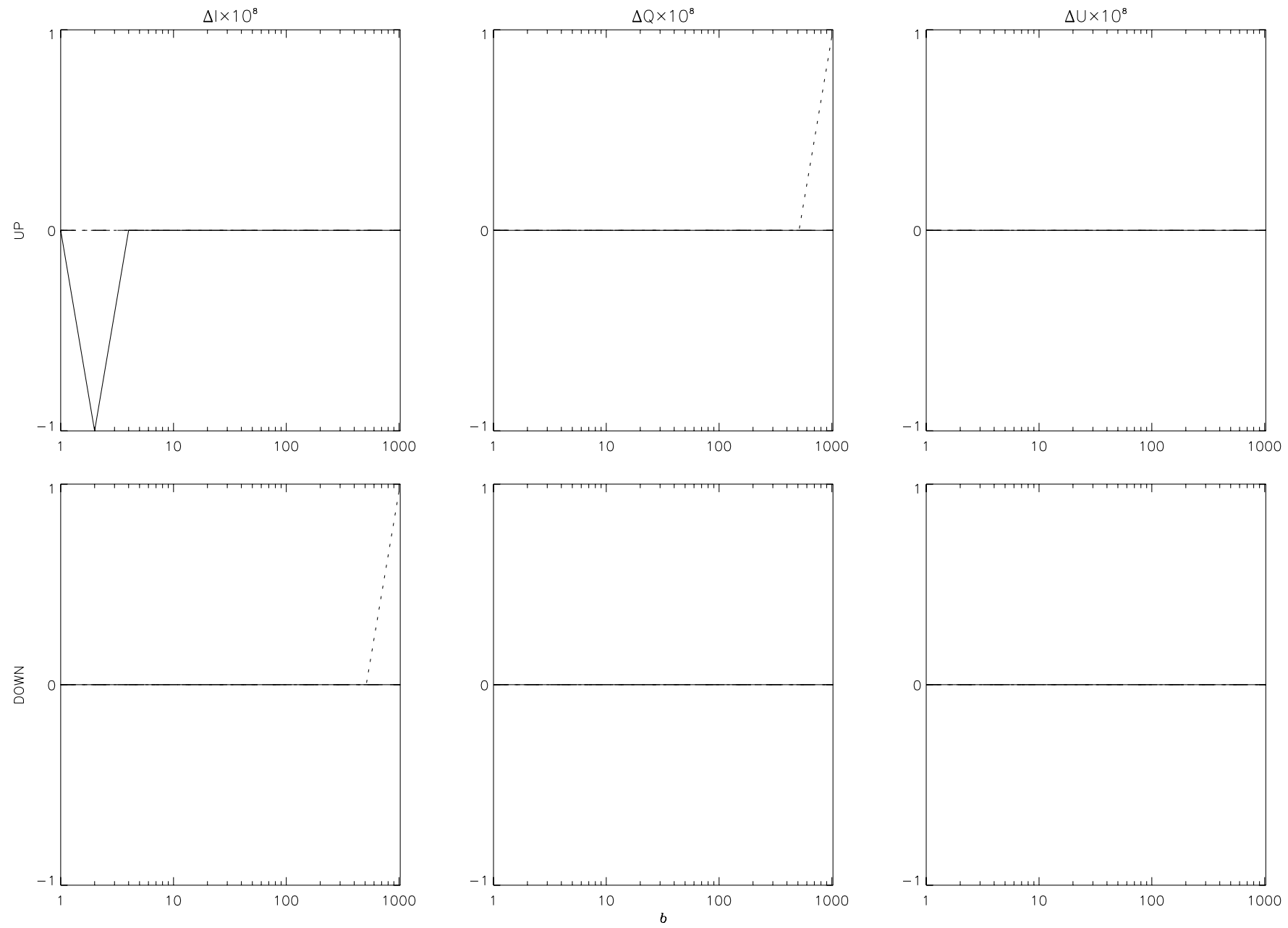

Figure 4. (Continued)

The unknowns in Equations (A4) can be calculated using the following expressions:

$$
\begin{aligned}
& q=2 \frac{4\left(A_{1}+2 \alpha_{1}\right)-3\left(A_{0} \alpha_{1}+\alpha_{0} A_{1}\right)}{3\left(A_{1}^{2}+2 \alpha_{1}^{2}\right)} \\
& c=\frac{8\left(A_{1}-\alpha_{1}\right)+3\left(2 \alpha_{1} \alpha_{0}-A_{1} A_{0}\right)}{3\left(A_{1}^{2}+2 \alpha_{1}^{2}\right)},
\end{aligned}
$$

where $A_{n}$ and $\alpha_{n}$ are moments of order $n$ of $H_{r}$ and $H_{l}$, respectively.

\section{APPENDIX B}

\section{CALCULATION OF $M$ - AND $H$-FUNCTIONS}

For the azimuth-dependent Fourier terms in Equations (8) we require the $M$-functions:

$$
M^{n}\left(\mu, \mu_{0}\right)=H^{n}(\mu) H^{n}\left(\mu_{0}\right) \quad(n=1,2),
$$

where $H^{1}$ and $H^{2}$ have the characteristic functions $\Psi^{1}$ and $\Psi^{2}$, respectively:

$$
\begin{gathered}
\Psi^{1}(\mu)=\frac{3}{8}\left(1-\mu^{2}\right)\left(1+2 \mu^{2}\right) \\
\Psi^{2}(\mu)=\frac{3}{16}\left(1+\mu^{2}\right)^{2} .
\end{gathered}
$$

Equation (A1) suggests that the $H$-functions need to be calculated by an iterative process. $H_{l}$ has a conservative characteristic function:

$$
\int_{0}^{1} \Psi_{l}(\mu) d \mu=\frac{1}{2} .
$$

For this case, we use the following alternative form of Equation (A1) (Chandrasekhar 1950):

$$
\frac{1}{H(\mu)}=\int_{0}^{1} \frac{\mu^{\prime} \Psi\left(\mu^{\prime}\right) H\left(\mu^{\prime}\right)}{\mu+\mu^{\prime}} d \mu^{\prime} .
$$

The reason for using Equation (B4) is that it facilitates convergence of the iterative procedure (Bosma \& De Rooij 1983).

Equation (B4) is solved using a minor modification of a procedure suggested by Bosma \& De Rooij (1983). We start with an initial guess $h^{(0)}(\mu)$, which we choose to be identically unity for all values of $\mu$. Inserting this value into the integral on the RHS of Equation (B4) gives a first iterate value. For the next guess, we use the average of this iterate and the previous guess. We repeat the above procedure until the sum over all quadrature angles of the difference between the current iterate and the previous iterate is less than a convergence threshold, which we set at $10^{-12}$. A final application of Equation (B4) yields the values at the angles of interest. This is essentially the same as method (i) of Bosma \& De Rooij (1983); the only difference is in the convergence criterion. Bosma \& De Rooij (1983) stop the iterations when the maximum difference between the current 
iterate and the previous iterate over all quadrature angles is less than the convergence threshold.

For all the other $H$-functions, we use another form of Equation (A1):

$$
\frac{1}{H(\mu)}=1-\mu \int_{0}^{1} \frac{\Psi\left(\mu^{\prime}\right) H\left(\mu^{\prime}\right)}{\mu+\mu^{\prime}} d \mu^{\prime},
$$

which is then solved by straightforward iteration. Equation (B5) follows from Equation (A1) by dividing both sides by $H(\mu)$. Note that Equation (B4) does not converge for straightforward iteration. Furthermore, if we compare Equation (B4) with the iterative procedure suggested by Bosma \& De Rooij (1983) and Equation (B5) with straightforward iteration, the latter converges roughly twice as fast.

\section{APPENDIX C}

\section{RELATION BETWEEN H-MATRIX AND $H$-FUNCTIONS}

The azimuth-independent component of the reflection matrix, $\mathbf{R}^{0}\left(\mu, \mu_{0}\right)$, at the top of a semi-infinite atmosphere can also be expressed in terms of the H-matrix (De Rooij et al. 1989):

$$
\mathbf{R}^{0}\left(\mu, \mu_{0}\right)=\frac{1}{4\left(\mu+\mu_{0}\right)} \mathbf{D}(\mu) \mathbf{H}(\mu) \mathbf{H}^{T}\left(\mu_{0}\right) \mathbf{D}^{T}\left(\mu_{0}\right)
$$

where:

$$
\mathbf{D}(\mu)=\left(\begin{array}{cc}
\frac{1}{4}\left(1-3 \mu^{2}\right) \sqrt{2} & 1 \\
\frac{3}{4}\left(1-\mu^{2}\right) \sqrt{2} & 0
\end{array}\right) .
$$

To find how $H_{l}$ and $H_{r}$ are related to the $\mathbf{H}$-matrix, we proceed as follows. First, we compute the azimuth-independent Fourier terms (Hovenier et al. 2004):

$$
\left(\begin{array}{l}
I^{0}\left(0 ; \mu, \mu_{0}\right) \\
Q^{0}\left(0 ; \mu, \mu_{0}\right)
\end{array}\right)=\mu_{0} \mathbf{R}^{0}\left(\mu, \mu_{0}\right) F_{0}\left(\begin{array}{l}
1 \\
0
\end{array}\right)
$$

where we have suppressed the subscript $\infty$ on the LHS.

From Equations (6a) and (6b) we obtain

$$
\left(\begin{array}{l}
I^{0}\left(0 ; \mu, \mu_{0}\right) \\
Q^{0}\left(0 ; \mu, \mu_{0}\right)
\end{array}\right)=\left(\begin{array}{cc}
1 & 1 \\
1 & -1
\end{array}\right)\left(\begin{array}{l}
I_{I}^{0}\left(0 ; \mu, \mu_{0}\right) \\
I_{r}^{0}\left(0 ; \mu, \mu_{0}\right)
\end{array}\right)
$$

and Equation (C3) becomes

$$
\left(\begin{array}{cc}
1 & 1 \\
1 & -1
\end{array}\right)\left(\begin{array}{l}
I_{I}^{0}\left(0 ; \mu, \mu_{0}\right) \\
I_{r}^{0}\left(0 ; \mu, \mu_{0}\right)
\end{array}\right)=\frac{1}{2} \mu_{0} \mathbf{R}^{0}\left(\mu, \mu_{0}\right) F_{0}\left(\begin{array}{cc}
1 & 1 \\
1 & -1
\end{array}\right)\left(\begin{array}{l}
1 \\
1
\end{array}\right) .
$$

Pre-multiplying both sides by $(1 / 2)\left(\begin{array}{cc}1 & 1 \\ 1 & -1\end{array}\right)$ gives

$$
\left(\begin{array}{l}
I_{I}^{0}\left(0 ; \mu, \mu_{0}\right) \\
I_{r}^{0}\left(0 ; \mu, \mu_{0}\right)
\end{array}\right)=\mu_{0} \frac{1}{4}\left(\begin{array}{cc}
1 & 1 \\
1 & -1
\end{array}\right) \mathbf{R}^{0}\left(\mu, \mu_{0}\right) F_{0}\left(\begin{array}{cc}
1 & 1 \\
1 & -1
\end{array}\right)\left(\begin{array}{l}
1 \\
1
\end{array}\right) .
$$

Comparing Equations (8a) and (C6), we obtain

$$
\frac{3}{8\left(\mu+\mu_{0}\right)} \mathbf{A}(\mu) \mathbf{A}^{T}\left(\mu_{0}\right)=\left(\begin{array}{cc}
1 & 1 \\
1 & -1
\end{array}\right) \mathbf{R}^{0}\left(\mu, \mu_{0}\right)\left(\begin{array}{cc}
1 & 1 \\
1 & -1
\end{array}\right) .
$$

From Equations $(\mathrm{C} 1)$ and $(\mathrm{C} 7)$, we now obtain a relationship between the $H_{l}$ and $H_{r}$ functions of Chandrasekhar (1950) and the H-matrix of De Rooij et al. (1989):

$$
\frac{3}{2} \mathbf{A}(\mu) \mathbf{A}^{T}\left(\mu_{0}\right)=\left(\begin{array}{cc}
1 & 1 \\
1 & -1
\end{array}\right) \mathbf{B}(\mu) \mathbf{B}^{T}\left(\mu_{0}\right)\left(\begin{array}{cc}
1 & 1 \\
1 & -1
\end{array}\right),
$$

where:

$$
\mathbf{B}(\mu)=\mathbf{D}(\mu) \mathbf{H}(\mu) .
$$

An even nicer form of the above relation is as follows:

$$
\mathbf{A}(\mu) \mathbf{A}^{T}\left(\mu_{0}\right)=\frac{2}{3} \mathbf{C}(\mu) \mathbf{C}^{T}\left(\mu_{0}\right),
$$

where

$$
\mathbf{C}(\mu)=\left(\begin{array}{cc}
1 & 1 \\
1 & -1
\end{array}\right) \mathbf{D}(\mu) \mathbf{H}(\mu)
$$

\section{REFERENCES}

Adams, C. N., \& Kattawar, G. W. 1970, J. Quant. Spectrosc. Radiat. Transfer, 10,341

Bosma, P. B., \& De Rooij, W. A. 1983, A\&A, 126, 283

Buenzli, E., \& Schmid, H. M. 2009, A\&A, 504, 259

Busbridge, I. W. 1960, The Mathematics of Radiative Transfer (Cambridge: Cambridge Univ. Press)

Chandrasekhar, S. 1950, Radiative Transfer (Oxford: Clarendon Press)

Chandrasekhar, S., \& Elbert, D. D. 1954, Trans. American Philos. Soc., 44, 643

Coffeen, D. L., \& Hansen, J. E. 1974, in Planets, Stars and Nebulae Studied with Photopolarimetry, ed. T. Gehrels (Tucson, AZ: Univ. Arizona Press), 518

Coulson, K. L., Dave, J. V., \& Sekera, Z. 1960, Tables Related to Radiation Emerging from a Planetary Atmosphere with Rayleigh Scattering (Berkeley: Univ. California Press)

Dave, J. V., \& Furukawa, P. M. 1966, J. Opt. Soc. Am., 56, 394

de Haan, J. F., Bosma, P. B., \& Hovenier, J. W. 1987, A\&A, 183, 371

De Rooij, W. A. 1985, PhD thesis, Free Univ., Amsterdam

De Rooij, W. A., Bosma, P. B., \& van Hooff, J. P. C. 1989, A\&A, 226, 347

Domke, H. 1978, Astron. Nachr., 299, 95

Fymat, A. L., \& Abhyankar, K. D. 1971, J. Geophys. Res. C, 76, 732

Hansen, J. E., \& Hovenier, J. W. 1974, J. Atmos. Sci., 31, 1137

Hovenier, J. W. 1970, A\&A, 7, 86

Hovenier, J. W., \& de Haan, J. F. 1985, A\&A, 146, 185

Hovenier, J. W., \& Stam, D. M. 2007, J. Quant. Spectrosc. Radiat. Transfer, 107,83

Hovenier, J. W., \& van der Mee, C. V. M. 1983, A\&A, 128, 1

Hovenier, J. W., van der Mee, C. V. M., \& Domke, H. 2004, Transfer of Polarized Light in Planetary Atmospheres (Dordrecht: Kluwer)

Joos, F., \& Schmid, H. M. 2007, A\&A, 463, 1201

Lyot, B. 1929, Ann. Obs. Paris (Meudon), 8, 1 (Engl. Transl., NASA TT F-187)

Natraj, V., Li, K.-F., \& Yung, Y. L. 2009, ApJ, 691, 1909

Schmid, H. M., Joos, F., \& Tschan, D. 2006a, A\&A, 452, 657

Schmid, H. M., Beuzit, J.-L., Feldt, M., et al. 2006b, in IAU Colloq. 200, Direct Imaging of Exoplanets: Science \& Techniques, ed. C. Aime \& F. Vakili (Cambridge: Cambridge Univ. Press), 165

Sekera, Z., \& Blanch, G. 1952, Tables Relating to Rayleigh Scattering of Light in the Atmosphere, Sci. Rep. 3, Contr. AF 19(122)-239 (Cambridge: Air Force Cambridge Res. Center)

Sekera, Z., \& Kahle, A. B. 1966, Scattering Functions for Rayleigh Atmospheres of Arbitrary Thickness, Rep. R-452-PR (Santa Monica: The Rand Corporation)

Siewert, C. E. 2000, J. Quant. Spectrosc. Radiat. Transfer, 64, 227

Spurr, R. J. D. 2006, J. Quant. Spectrosc. Radiat. Transfer, 102, 316

Stam, D. M. 2008, A\&A, 482, 989

Stam, D. M., De Rooij, W. A., Cornet, G., \& Hovenier, J. W. 2006, A\&A, 452, 669

Stam, D. M., Hovenier, J. W., \& Waters, L. B. F. M. 2004, A\&A, 428, 663

Stokes, G. G. 1852, TCaPS, 9, 399

van de Hulst, H. C. 1957, Light Scattering by Small Particles (New York: Wiley) van de Hulst, H. C. 1980, Multiple Light Scattering (New York: Academic)

Wauben, W. M. F., de Haan, J. F., \& Hovenier, J. W. 1994, A\&A, 281, 258

West, R. A., \& Smith, P. H. 1991, Icarus, 90, 330 